\title{
1 Intracellular metabolic circuits shape inter-species microbial
}

\section{2 interactions}

6 Ali R. Zomorrodi ${ }^{1}$ and Daniel Segrè̀,2,3*

$8 \quad{ }^{1}$ Bioinformatics Program, Boston University, Boston, MA 02215

9 2Department of Biology, Boston University, Boston, MA 02215

10 32Department of Biomedical Engineering, Boston University, Boston, MA 02215

11

12

$13{ }^{*}$ Corresponding author:

14 Email: dsegre@bu.edu

15 Phone: 617-358-2301

16 Fax: 617-353-4814

17

18

19

20

21

22

23

24

25

26

27 
1 Metabolite exchanges in microbial communities give rise to ecological

2 interaction networks that influence ecosystem diversity and stability ${ }^{1-6}$. These

3 exchanges depend on complex intracellular pathways thus raising the

4 question of whether ecological interactions are inferable from genomes. We

5 address these questions by integrating genome-scale models of metabolism ${ }^{7}$,

6 to compute the fitness of interacting microbes, with evolutionary game theory,

7 which uses these fitness values to infer evolutionarily stable interactions in

8 multi-species microbial "games". After validating our approach using data on

9 sucrose hydrolysis by $S$. cerevisiae, we performed over 80,000 in silico

10 experiments to evaluate the rise of unidirectional and cross-feeding metabolic

11 dependencies in populations of Escherichia coli secreting 189 amino acid

12 pairs. We found that, despite the diversity of exchanged amino acids, most

13 pairs conform to general patterns of inter-species interactions. However,

14 several amino acid pairs deviate from these patterns due to pleiotropy and

15 epistasis in metabolic pathways. To better understand the emergence of cross-

16 feeding, we performed in silico invasion experiments and found possible

17 evolutionary paths that could lead to such association. Overall, our study

18 provides mechanistic insights into the rise of evolutionarily stable

19 interdependencies, with important implications for biomedicine and

20 microbiome engineering ${ }^{8-10}$. 
1 Obligate dependencies among microorganisms, through the exchange of essential

2 metabolites have been hypothesized to be ubiquitous in microbial ecosystems ${ }^{1}$.

3 Similar interactions have also been engineered in laboratory systems, mainly based

4 on genetically induced auxotrophies 3-6,11,12. However, the evolutionary rise and

5 maintenance of these interactions constitutes an unresolved puzzle, since genotypes

6 that do not produce a given costly metabolite may have a selective advantage over

7 producers. One theory, known as the Black Queen Hypothesis 13, suggests that

8 metabolic dependencies could arise through adaptive gene loss: organisms that lose

9 the capacity to produce a costly compound (non-producers) will have a selective

10 advantage over organisms that produce and inevitably leak that compound

11 (producers). This could give rise to an obligate dependency of non-producers on

12 producers 13 , or, in the case of more than one leaky function, to obligate cross-

13 feeding (bidirectional dependency) ${ }^{14}$. However, little is known about the conditions

14 under which these dependencies would be established.

16 A limited number of theoretical studies have recently explored this question using

17 ecological models 15-18, evolutionary game theory (see 19-21 for comprehensive

18 reviews) and concepts from economics 22 . While these approaches have provided

19 valuable phenomenological insight into the general principles of metabolic

20 interdependencies, they generally do not take into account the specific details of the

21 organisms, pathways and molecules involved: behind the production, leakiness, and

22 utilization of each metabolite, is a complex network of biochemical reactions, which

23 may significantly vary from organism to organism, and across different conditions

24 and exchanged metabolites. A powerful avenue to address this gap is the use of

25 systems biology methods, such as stoichiometric models of metabolism ${ }^{7}$. These

26 models take into account the full metabolic circuitry of a cell and provide

27 quantitative predictions of its growth capacity and metabolic fluxes. Recent work

28 has started applying these approaches to model microbial communities (see ${ }^{23}$ for a

29 recent review) and to study the evolution of adaptive diversification in long-term

30 evolutionary experiments 24 . However, a systematic analysis of the underlying

31 mechanisms and patterns of possible equilibrium states of inter-species interactions 
1 as a function of the leakiness of different metabolites is still unexplored. Here we

2 propose a new hybrid modeling approach that combines the theoretical insight of

3 evolutionary game theory with the organism-specific detailed analysis of cell-wide

4 metabolic networks. We demonstrate how this strategy allows us to map the

5 landscape of possible inter-species interactions, for which genome-scale models

6 provide unique mechanistic insight.

8 Our mechanistic evolutionary game theory approach uses estimates of microbial

9 fitness (payoff), based on genome-scale metabolic models, (forming the "payoff

10 matrix" of the game). These payoffs are subsequently utilized to compute the Nash

11 equilibria of "microbial interaction games" (see Methods). A Nash equilibrium is a

12 central concept in game theory, defined as a state where no player can increase its

13 payoff by a unilateral change of strategy. These payoffs also allows us to determine

14 which of the identified Nash equilibria are evolutionarily stable by modeling the

15 dynamics of genotype frequencies ${ }^{25}$ (see Figure 1 and Methods). As a proof-of-

16 concept of our approach, we modeled the yeast sucrose hydrolysis system ${ }^{26}$ : when

17 growing on sucrose, S. cerevisiae produces the surface enzyme invertase (encoded

18 by the suc2 gene), hydrolyzing sucrose into glucose and fructose, part of which serve

19 as a public good. Since invertase production is metabolically costly, a mutant strain,

20 which has lost its suc2 gene, may emerge (see Figure 2A). Our simulations (based on

21 the iAZ900 yeast metabolic model ${ }^{27}$ ) reproduced the three types of equilibria

22 observed experimentally ${ }^{26}$, i.e. Prisoners' Dilemma (non-producers dominate and

23 the community collapses), Mutually Beneficial game (producers dominate), and

24 Snowdrift game (producers and non-producers coexist) (see Figure 2B-2F, and

25 supplementary text for details).

27 We next sought to characterize the landscape of possible ecological interactions in a

28 large set of strains and exchanged metabolites. In particular, we explored the

29 evolution of metabolic dependencies mediated by the leaky production of individual

30 amino acids in E. coli and asked how the evolution of these dependencies varies

31 across the 20 amino acids and different leakiness levels. Here, a prototrophic wild- 
1 type (WT, producer), leaking a given amino acid could interact with a mutant strain

2 (MT, non-producer) lacking the gene(s) for the biosynthesis of that amino acid (see

3 Figure 2G). The iJ01366 genome scale model of E. coli ${ }^{28}$ was used to construct in

4 silico producer and non-producer strains, and to explore what equilibria can be

5 established for a given leakiness level. As shown in Figure 2H, Prisoner's Dilemma,

6 Mutually Beneficial and Snowdrift outcomes are the major possible equilibria,

7 similar to the yeast system (Figure 2B) (with the amino acid cost replacing the

8 sucrose hydrolysis cost). However, a more complex pattern is observed here for the

9 Mutually Beneficial region (see supplementary text for details), highlighting the

10 organism- and product-specific nature of our model. In addition, one can observe

11 that, for each amino acid, there is a threshold for leakiness level above which non-

12 producer mutants (MT) dominate, leading to community collapse (i.e., Prisoner's

13 Dilemma) (Figure 3H). Thus, we may expect the amino acids secretion levels in $E$.

14 coli to lie below this threshold. Interestingly, the average of multiple published

15 amino acid secretion datasets (see Figure 3J) displays a consistent trend of leakiness

16 levels decreasing with increasing cost. To assess the evolutionary stability of above

17 Nash equilibria we performed in silico invasion experiments, where a resident

18 population of WT is invaded by a low frequency MT (and vice versa). This analysis

19 showed that the equilibrium frequencies of WT and MT are independent of their

20 initial frequencies (see Figure 3I). This had been theoretically ${ }^{14}$ and experimentally

2114,29 suggested to stem from the negative frequency dependence of fitness. In

22 addition to recapitulating this pattern, our analysis provides a quantitative

23 prediction of the selection coefficients of the 20 amino acids. As shown in Figure 3K,

24 the predicted selection coefficients display reasonable agreement with previous

25 experimental reports for E. coli ${ }^{29}$. This establishes a nontrivial quantitative link

26 between metabolic stoichiometry and important ecological parameters.

28 We further extended our analysis to map the landscape of ecological interactions in

29 communities with two leaky amino acids. Under what conditions would the

30 increased number of exchangeable metabolites give rise to more complex inter-

31 species interdependencies, such as reciprocal exchanges? Four different genotypes 
1 are possible in this case (see Figure 3A): a prototrophic genotype that produces and

2 leaks two amino acids (i.e., a full producer, denoted as '11'), two partial producer

3 mutants each lacking the gene(s) for the biosynthesis of one amino acid (denoted as

4 ' 01 ' and ' 10 '), and a no-producer mutant strain lacking the biosynthesis genes for

5 both amino acids (denoted as '00'). In a community composed of all these four

6 genotypes, different types of interactions are possible. Here we focus on pairwise

7 interactions, such as cross-feeding, [01, 10] and unidirectional dependency, [00, 11],

8 though higher-order interactions among three or four genotypes (e.g., [00, 01, 10]

9 and $[00,01,10,11])$ are possible as well. We next systematically computed all Nash

10 equilibria, at varying leakiness levels, for 189 amino acid pairs, corresponding to all

11 possible pairs of 20 amino acids (see Figure 3B) except for one, namely the (alanine,

12 isoleucine) pair, because the 00 genotype for this pair is auxotrophic for a third

13 amino acid, valine. (see supplementary Figures S2 to S7 for higher order

14 interactions' equilibria).

16 As shown in Figure 3B, a wide spectrum of equilibria ensues across different amino

17 acid pairs and across different leakiness levels (e.g., see Figures 4C and 4D).

18 Interestingly, despite the diversity of strains and exchanged metabolites, a majority

19 of pairs are found to conform to general ecological patterns: for example, 139 (out of

20189 , or $73.5 \%$ ) of amino acid pairs display a qualitatively identical region of

21 leakiness levels (green regions in Figures 4B,C), where a unidirectional association,

$22[00,11]$, and cross-feeding, $[01,10]$, simultaneously emerge as Nash equilibria. The

23 leakiness levels leading to this mixed equilibrium are the ones for which the full

24 producer (i.e., 11 genotype) can still sustain growth. We refer to this region as

25 sustainable leakiness region (see Methods). This is analogous to the maximum

26 leakiness level in the study of individual amino acid secretions (Figure 2H): any

27 leakiness levels for the two amino acids that lie outside this region would be

28 expected to lead to extinction of the wild-type.

30 Another feature common to several amino acid pairs is the existence of a region in

31 the leakiness plane (shown by red in Figure 3C) where cross-feeding (i.e., [01, 10]) 
1 is the only viable association, since excessive leakiness makes the full producer, 11,

2 non-viable. This region is contiguous to the green region (with $[00,11]$ and $[01,10]$

3 as Nash equilibria). One interesting aspect of this configuration is that cross-feeding

4 could initially ensue in the green region, coexisting with $[00,11]$ genotypes, and

5 gradually move towards the red region (by evolving increased leakiness through

6 selective advantage relative to the ancestor 11 ), leading to obligate cross-feeding as

7 the only viable association.

9 In addition to exploring the landscape of Nash equilibria across different leakiness

10 levels, it is interesting to ask whether the details of the biochemical networks

11 underlying the genome-scale metabolic model predictions matter, and whether they

12 provide direct explanatory power at the ecological level. Notably, for 50 amino acid

13 pairs, the region of sustainable leakiness levels does not conform to the general

14 pattern described above. For some pairs this region is partitioned into a number of

15 sub-regions each corresponding to a different equilibrium (e.g. see Figure 3D) while

16 in some extreme cases this entire region corresponds to a single Nash equilibrium,

17 e.g., for (arginine, glutamate) and (glycine, threonine) (see below).

19 For the first anomalous pair, (arginine, glutamate), Nash equilibria in the 20 sustainable leakiness region includes $[10,11]$ and $[00,11]$, but not the cross-feeding

21 state [01, 10] (see Figure 3E). Inspection of the biosynthesis pathway of arginine 22 and glutamate in E. coli revealed that glutamate is required for the production of 23 ornithine, which serves as an essential precursor for the biosynthesis of arginine.

24 This implies that a mutant strain lacking the biosynthesis pathways for glutamate 25 (i.e., strain 10) will not be able to synthesize and leak arginine, thus acting like a 00 26 genotype and preventing the occurrence of cross-feeding. This observation is 27 consistent with a previous report on the inability of arginine and glutamate 28 auxotrophic mutant strains to grow in a co-culture under the minimal medium ${ }^{3}$.

29 This effect is due to a pleiotropic metabolic gene (i.e. a gene whose modification

30 affects more than one metabolic phenotype), and illustrates how core biochemistry 31 can impact ecological interactions. 
2 A more complex scenario occurs for the (glycine, threonine) pair, where cross-

3 feeding, [01, 10], is the only Nash equilibrium that emerges in the sustainable

4 leakiness region (see Figure 3F). What prevents [00, 11] from being a Nash

5 equilibrium? One of the conditions for $[00,11]$ to be a Nash equilibrium is that, in

6 presence of 11, the fitness of the non-producer (00) should be higher than any of the

7 partial producers (01 and 10) (as one would intuitively expect, because 00 does not

8 incur the production cost of the two amino acids). In this case, however, it turns out

9 that 00 is less fit than $10\left(t h r^{-}\right.$mutant, see a sample payoff matrix in supplementary

10 Figure S8) thereby preventing [00, 11] from being a Nash equilibrium. This

11 anomalous effect is due to the fact that the concurrent removal of glycine and

12 threonine biosynthesis genes (in 00) will lead to a reduction in the capacity to

13 produce other essential biomass components (such as serine). Interestingly, this is a

14 case of diminishing-return (or "negative") epistasis, in which the effect of the double

15 mutation (00) is less severe than expected based on the two single mutations (01

16 and 10). Negative epistatic interactions preventing the appearance of $[00,11]$ as the

17 Nash equilibrium can be observed for 34 other amino acid pairs (see supplementary

18 Table S1). This pattern is supported by existing experimental reports showing that

19 epistasis correlates negatively with the expected fitness of multiple "genome

20 streamlining" mutations in E. coli, thereby causing diminishing returns ${ }^{30}$. These

21 results provide mechanistic insights into how epistatic interactions among

22 intracellular pathways can affect ecological interactions, a feature that cannot be

23 captured by abstract phenomenological models.

25 It is next interesting to ask whether our approach can shed light onto the possible 26 paths towards the rise of different interactions, especially cross-feeding. In the 27 landscapes of identified Nash equilibria (Figure 3B), cross feeding ([01, 10]) 28 emerges together with other equilibria (such as [00,11], e.g. in the green region in 29 Figure 3C). This raises the question of whether and under what conditions cross30 feeding would be evolutionarily stable. By performing a number of targeted in silico 31 invasion experiments (see Methods), we found that this depends strongly on the 
1 initial frequencies of the different genotypes (shown in Figure 3A). In particular, our

2 analysis demonstrates that cross-feeders (01 and 10) will go extinct if they invade

3 the full producer (11) in presence of the non-producer genotype (00) (see Figure

4 4A). However, the cross-feeders can subsist if 00 invades at a later stage, e.g. if it

5 arises from mutations in the partial producers (01 or 10) (see Figures $4 \mathrm{~B}$ and $4 \mathrm{C}$ for

6 two such mechanisms). An example of the latter scenario is the two-step process

7 hypothesized in 14: first, the biosynthetic capacity for one amino acid is lost, e.g.

8 resulting in a 01 genotype, which could equilibrate and co-exist with 11 (see Figures

$92 \mathrm{G}-2 \mathrm{I})$. In the second step, either 01 or 11 may lose their capacity for producing the

10 second amino acid (because the other strain can compensate), giving rise to 00 and

1110 genotypes, respectively (see Figure 4B). Here, we quantitatively explored this

12 hypothesis by assessing the evolutionary dynamics for the second step, assuming

13 that equilibration of the first step has already occurred. As shown in Figure 4B, the

14 prototrophic (11) and no-producer (00) genotypes always survive in this

15 competition, while cross-feeders evolutionarily emerge only at high leakiness levels.

16 Further in silico invasion experiments demonstrate that established cross-feeding

17 pairs tend to be resistant to invasion by non-producers (see supplementary Figure

18 S10) and by prototrophs (see supplementary Figure S11). Thus, once established,

19 obligate mutual metabolic exchange could be evolutionarily stable against invasion

20 by other genotypes, even in a homogenous environment, consistent with previous

21 experimental reports ${ }^{31}$. Notably this stability against invasions is dependent on the

22 specific metabolites exchanged and on the level of metabolic exchange (leakiness).

24 We demonstrated here that by adding new layers of details to abstract theoretical

25 ecology models, we can reveal how intracellular molecular mechanisms (including

26 pleiotropy and epistasis of metabolic enzyme genes) lead to the rise of ecological

27 interactions. The analysis we presented spans over 80,000 in silico experiments

28 (across 189 amino acid pairs and 441 leakiness level combinations), which is

29 beyond the current experimental capabilities. This study provides a guideline for

30 the design of future targeted experiments, e.g. built upon previously established

31 synthetic communities 3-6,11. For example, our computational results could be used 
1 to suggest choices of metabolite pairs and ranges of engineered leakiness levels that

2 lead to the establishment of a specific inter-dependency, e.g., unidirectional vs.,

3 cross-feeding. In addition, our study offers a basis for better understanding of

4 metabolic interdependencies in natural microbial communities, such as those in the

5 human gut microbiota ${ }^{8-10}$. From the perspective of biotechnological applications,

6 our approach lays the foundation for proactively incorporating evolutionary

7 concepts in the de novo design of synthetic microbial consortia that are resistant to

8 invasion by competing strategies ${ }^{23}$.

\section{Methods}

10 Background on evolutionary game theory. Evolutionary game theory is the

11 application of classical game theory to model the evolutionary dynamics of mixed

12 populations. Modeling microbial communities with evolutionary game theory

13 involves two steps: (i) Considering all pairwise interactions among genotypes and

14 estimating the payoff (fitness) of a microbial player $k$ upon interacting with a

15 partner $k^{\prime}$. Higher order interactions can be similarly defined; These estimated

16 payoffs are represented in the form of a matrix, referred to as payoff matrix. (ii)

17 Using the payoff matrix to identify the Nash equilibrium(s) - a fundamental concept

18 in game theory defined as a state where no player has an incentive to unilaterally

19 change its current strategy, because it cannot improve its payoff by doing so. An

20 evolutionary stable strategy is a similar concept in evolutionary game theory: It is a

21 Nash equilibrium, which is evolutionarily stable, i.e., natural selection alone is

22 sufficient to prevent invasion by competing mutant strategies. Evolutionary stable

23 strategies can be found by modeling the evolutionary dynamics of the game using

24 the computed payoffs (see the following sections). In this text, by 'evolutionary

25 dynamics' we mean how the relative genotype abundances (frequencies, or

26 community structure) change over time following evolutionary game theory ${ }^{25}$, and

27 microbial ecology literature ${ }^{32}$. This aspect of evolutionary dynamics, as opposed to

28 the simulation of de novo mutations and subsequent selection processes (which

29 have been pursued in other studies ${ }^{33}$ ), is the main focus of our analysis. 
2 Background on Flux Balance Analysis (FBA). FBA (described in detail elsewhere,

3 e.g. ${ }^{34}$ ) is a linear optimization problem that uses genome-scale metabolic models to

4 make quantitative predictions about the cell's growth rate, intracellular reaction

5 fluxes and secretion rates of metabolites that are potentially excreted by the cell

6 under a given condition. In the most common formulation, this is achieved by

7 maximizing the flux of a pseudo-reaction called biomass reaction $\left(v_{\text {biomass }}\right)$ whose

8 reactants are precursors required for growth and whose flux is indicative of the

9 cell's growth capacity. This is subject to constraints imposing the steady-state mass

10 balance for each metabolite in the network (see Constraint 1 below) and lower and

11 upper bounds on reactions fluxes to impose the reversibility of reactions and uptake

12 and aeration conditions (see Constraint 2 below). The standard formulation of FBA

13 is as follows:

maximize $v_{\text {biomass }}$

subject to

$\begin{array}{ll}\sum_{j \in J} s_{i j} v_{j}=0, & \forall i \in I, \\ L B_{j} \leq v_{j} \leq U B_{j}, & \forall j \in J,\end{array}$

15 where, $I$ is the set of metabolites, $J$ is the set of reactions, $s_{I j}$ is the stoichiometric

16 coefficient of metabolite $i$ in reaction $j$ (known from the metabolic model), $L B_{j}$ and

$17 U B_{j}$ denote lower and upper bounds on flux of a reaction $j$ (provided as inputs),

18 respectively, and $v_{j}$ is the flux of a reaction $j$ (optimization variables).

20 Using genome-scale models and FBA to compute payoffs for interacting

21 microbes. We used FBA to provide organism-specific and genomically-informed

22 estimates of the payoffs upon specific pairwise interactions (see also Figure 1). For a

23 given pair of genotypes $k$ and $k^{\prime}$, we solve a separate FBA problem for $k$ and $k^{\prime}$. Each

24 FBA problem involves two new types of constraints added to the standard FBA: (i)

25 The first type of constraints pertains to implementing in silico the gene deletions 
1 that correspond to the genotype under consideration (see Equation 3 below). For

2 example, if genotype $k$ is auxotroph for lysine, this auxotrophy can be in principle

3 induced by knocking out gene lys , which codes for diaminopimelate decarboxylase.

4 (DAPC). In the FBA model, this gene deletion is simulated by setting $v_{D A P D C}=0$.

5 More complex gene-to-reaction mappings could lead to more complex set of

6 constraints. In cases where several different choices are possible for genes whose

7 deletion would induce a given auxotrophy, we select one. (ii) The second type of

8 constraints (Equations 4 and 5) simulates the exchange of metabolites between the

9 genotype under consideration and its partner(s). In our calculations, for any given

10 exchanged metabolite (e.g., amino acid or amino acids pair), we systematically

11 explore the effects of a range of possible leakiness levels. Thus in each in silico

12 experiment, the leakiness levels of amino acids secreted by each genotype are fixed

13 at a pre-specified value. Similarly, metabolites leaked by the partner are made

14 available to this genotype through appropriate upper bounds on import fluxes. The

15 optimal value of biomass flux obtained upon solving this FBA problem provides an

16 estimate of the growth rate of each genotype in a given pairwise interaction, which

17 we use as a proxy for its payoff. For example, the payoff of $k$ when facing $k^{\prime}\left(a_{k k^{\prime}}\right)$ is

$18 v_{\text {biomass }}^{k}$ and the payoff of $k^{\prime}$ when facing $k\left(a_{k^{\prime} k}\right)$ is $v_{\text {biomass. }}^{k^{\prime}}$. The general form of the

19 FBA formulation for each genotype is mathematically formulated as follows:

maximize $v_{\text {biomass }}$

subject to

$\sum_{j \in J} s_{i j} v_{j}=0, \quad \forall i \in I$,

$L B_{j} \leq v_{j} \leq U B_{j}, \quad \forall j \in J$,

$v_{j}=0, \quad \forall j \in J^{\text {mutant }}$

$v_{E X_{-} i(e)} \geq-u_{i}, \quad \forall i \in I^{\text {auxotroph }}$

$v_{E X_{-} i(e)} \geq e_{i}, \quad \forall i \in I^{l e a k y}$

21 where, Jutation denotes the set of reactions corresponding to specific gene

22 mutations for the genotype under consideration. In addition, $I^{\text {auxotroph }} \subset I$ 
1 represents the set of metabolites that this genotype is auxotroph for, but that are

2 provided by other genotypes, and $I^{\text {leaky }} \subset I$ is the set of leaky (secreted)

3 metabolites by the genotype under consideration. $v_{E X_{-} i(e)}$ denotes the flux of

4 exchange reaction for a metabolite $i . u_{i}>0$ and $e_{i}>0$ denote the pre-specified net

5 uptake and export flux of a metabolite $i$, respectively. Input parameters for this FBA

6 problem include: (i) The list of metabolites that each genotype is leaking and those

7 available for uptake from the partner genotype in a pairwise (or higher order)

8 interaction, (ii) The leakiness level of exchanged metabolites, and (iii) the net uptake

9 and export fluxes of exchanged metabolites (i.e., $e_{i}$ and $u_{I}$ ) calculated based on the

10 fixed leakiness levels and the specific interacting genotypes (see supplementary text

11 for details). Constraint (3) sets to zero the flux of reactions corresponding to the

12 specific gene mutations in the genotype under consideration. Constraints (4) allows

13 for the uptake of metabolites available from partner genotype(s) in a pairwise or

14 (higher-order) interaction. Constraint (5) requires the export of leaky metabolites at

15 the pre-specified level $e_{i}$. The payoff of the genotype under consideration is set to

16 the optimal value of the biomass flux, or to the death rate (a negative value), in the

17 case of an infeasible problem. An infeasible FBA problem may occur due the lack of

18 enough carbon source to satisfy maintenance ATP requirements in the model or due

19 to imposing a high level of leakiness for a leaked metabolite. Imposed leakiness level

20 causing this infeasibility are referred to 'unsustainable leakiness levels'. The details

21 of specific formulations for the presented case studies with $S$. cerevisiae and E. coli

22 are given in the supplementary text. Additional environmental/strategic/genetic

23 conditions can be incorporated through the addition of appropriately defined

24 constraints. In addition, one can use a different objective function (e.g., the

25 minimization of metabolic adjustment ${ }^{35}$ ) or other constraint-based community

26 modeling tools e.g., ${ }^{36,37}$ as an alternative.

28 Optimization-driven automated identification of the Nash equilibria. Upon

29 constructing the payoff matrix, as described above, one can identify the Nash

30 equilibrium(s) of the game. We developed an efficient optimization-based 
1 procedure called NashEq Finder to automate the identification of all pure strategy

2 Nash equilibria of a game given its payoff matrix. NashEq Finder is an integer linear

3 program (ILP), which relies on binary variables to decide on whether or not a

4 particular entry of the payoff matrix satisfies the conditions of a Nash equilibrium

5 (see supplementary text for a detailed formulation). This algorithm is able to

6 identify all possible Nash equilibria of a game with any number of players and thus

7 can be reliably used for the rapid identification of the equilibrium states of complex

8 communities.

10 Modeling the evolutionary dynamics at a genome-scale resolution. Following

11 standard approaches in evolutionary game theory we model evolutionary dynamics

12 using the Replicator Equation 25. In particular, to take into account interactions

13 higher than pairwise, we used an extended form of the classical Replicator equation

1425 . This equation predicts the changes in the relative abundance (frequencies) of

15 genotypes over time according to their reproductive fitness under the assumption of

16 a roughly constant population size ${ }^{25}$ (see supplementary text for the difference

17 between this equation and multi-species dynamics flux balance analysis ${ }^{38}$ ). Our

18 formulation, following ${ }^{39}$, can be expressed as follows:

$\frac{d x_{k}}{d t}=\left(f_{k}(\boldsymbol{x})-\phi(\boldsymbol{x})\right) x_{k}, \quad k=1,2, \ldots, K$,

$f_{k}(\boldsymbol{x})=\sum_{k^{\prime}=1}^{K} a_{k k^{\prime}} x_{k^{\prime}}+\sum_{k^{\prime}=1}^{K} \sum_{k^{\prime \prime}=1}^{K} a_{k k^{\prime} k^{\prime \prime}} x_{k^{\prime}} x_{k^{\prime \prime}}+\cdots, \quad k=1,2, \ldots, K$,

$\phi(x)=\sum_{k^{\prime}=1}^{K} f_{k^{\prime}}(\boldsymbol{x}) x_{k^{\prime}}$

19 Here, $K$ denotes the set of community members and $\boldsymbol{x}=\left[x_{1}, x_{2}, \ldots, x_{K}\right]^{T}$ is the

20 composition of the community with $x_{k}$ being the frequency of genotype $k . f_{k}(\boldsymbol{x})$ is

21 the average reproductive fitness of genotype $k$ that depends not just on other

22 genotypes it may encounter but also on their frequencies. $a_{k k^{\prime}}$ and $a_{k k^{\prime} k^{\prime \prime}}$ denote the

23 payoffs of genotype $k$ when encountering another genotype $k^{\prime}$ in a two-player game 
1 (i.e. pairwise interaction) or two other genotypes $k^{\prime}$ and $k^{\prime \prime}$ in a three-player game,

2 respectively. Finally, $\phi(\boldsymbol{x})$ is the average fitness of the entire community.

4 Here we use the replicator equation to perform targeted in silico invasion

5 experiments, in which a newly emerged low frequency genotype invades an existing

6 resident genotype. While such in silico experiments are based only on evolutionary

7 dynamics (modeled through the Replicator equation), future developments could

8 take into account additional aspects such as eco-evolutionary feedback $32,40-42$.

\section{References}

111 Zelezniak, A. et al. Metabolic dependencies drive species co-occurrence in diverse microbial communities. Proc Natl Acad Sci U S A 112, 6449-6454, doi:10.1073/pnas.1421834112 (2015).

2 Freilich, S. et al. Competitive and cooperative metabolic interactions in bacterial communities. Nat Commun 2, 589, doi:10.1038/ncomms1597 (2011).

3 Wintermute, E. H. \& Silver, P. A. Emergent cooperation in microbial metabolism. Mol Syst Biol 6, 407, doi:10.1038/msb.2010.66 (2010).

4 Mee, M. T., Collins, J. J., Church, G. M. \& Wang, H. H. Syntrophic exchange in synthetic microbial communities. Proc Natl Acad Sci U S A 111, E2149-2156, doi:10.1073/pnas.1405641111 (2014).

5 Shou, W., Ram, S. \& Vilar, J. M. Synthetic cooperation in engineered yeast populations. Proc Natl Acad Sci $U$ S A 104, 1877-1882, doi:10.1073/pnas.0610575104 (2007).

6 Hoek, T. A. et al. Resource Availability Modulates the Cooperative and Competitive Nature of a Microbial Cross-Feeding Mutualism. PLoS Biol 14, e1002540, doi:10.1371/journal.pbio.1002540 (2016).

7 Becker, S. A. et al. Quantitative prediction of cellular metabolism with constraint-based models: the COBRA Toolbox. Nat Protoc 2, 727-738, doi:10.1038/nprot.2007.99 (2007).

8 Rakoff-Nahoum, S., Foster, K. R. \& Comstock, L. E. The evolution of cooperation within the gut microbiota. Nature 533, 255-259, doi:10.1038/nature17626 (2016).

9 Waldor, M. K. et al. Where next for microbiome research? PLoS Biol 13, e1002050, doi:10.1371/journal.pbio.1002050 (2015).

10 Magnúsdóttir, S. et al. Generation of genome-scale metabolic reconstructions for 773 members of the human gut microbiota. Nat Biotechnol 35, 81-89, doi:10.1038/nbt.3703 (2017). 
111 Harcombe, W. Novel cooperation experimentally evolved between species.

Evolution 64, 2166-2172, doi:10.1111/j.1558-5646.2010.00959.x (2010). Zhang, X. \& Reed, J. L. Adaptive evolution of synthetic cooperating communities improves growth performance. PLoS One 9, e108297, doi:10.1371/journal.pone.0108297 (2014).

13 Morris, J. J., Lenski, R. E. \& Zinser, E. R. The Black Queen Hypothesis: evolution of dependencies through adaptive gene loss. MBio $\mathbf{3}$, doi:10.1128/mBio.00036-12 (2012).

14 Morris, J. J. Black Queen evolution: the role of leakiness in structuring microbial communities. Trends Genet 31, 475-482, doi:10.1016/j.tig.2015.05.004 (2015).

15 Oliveira, N. M., Niehus, R. \& Foster, K. R. Evolutionary limits to cooperation in microbial communities. Proc Natl Acad Sci US A 111, 17941-17946, doi:10.1073/pnas.1412673111 (2014).

16 Mas, A., Jamshidi, S., Lagadeuc, Y., Eveillard, D. \& Vandenkoornhuyse, P. Beyond the Black Queen Hypothesis. ISME J, doi:10.1038/ismej.2016.22 (2016).

17 Estrela, S., Morris, J. J. \& Kerr, B. Private benefits and metabolic conflicts shape the emergence of microbial interdependencies. Environ Microbiol 18, 1415-1427, doi:10.1111/1462-2920.13028 (2016).

18 Germerodt, S. et al. Pervasive Selection for Cooperative Cross-Feeding in Bacterial Communities. PLoS Comput Biol 12, e1004986, doi:10.1371/journal.pcbi.1004986 (2016).

19 Frey, E. Evolutionary game theory: Theoretical concepts and applications to microbial communities. Physica a-Statistical Mechanics and Its Applications 389, 4265-4298, doi:10.1016/j.physa.2010.02.047 (2010).

20 Hummert, S. et al. Evolutionary game theory: cells as players. Mol Biosyst 10, 3044-3065, doi:10.1039/c3mb70602h (2014).

21 Blaser, M. J. \& Kirschner, D. The equilibria that allow bacterial persistence in human hosts. Nature 449, 843-849, doi:10.1038/nature06198 (2007).

22 Tasoff, J., Mee, M. T. \& Wang, H. H. An Economic Framework of Microbial Trade. PLoS One 10, e0132907, doi:10.1371/journal.pone.0132907 (2015).

23 Zomorrodi, A. R. \& Segrè, D. Synthetic Ecology of Microbes: Mathematical Models and Applications. J Mol Biol, doi:10.1016/j.jmb.2015.10.019 (2015).

24 Großkopf, T. et al. Metabolic modelling in a dynamic evolutionary framework predicts adaptive diversification of bacteria in a long-term evolution experiment. BMC Evol Biol 16, 163, doi:10.1186/s12862-016-0733-x (2016).

25 Nowak, M. A. Evolutionary dynamics : exploring the equations of life. (Belknap Press of Harvard University Press, 2006).

26 Gore, J., Youk, H. \& van Oudenaarden, A. Snowdrift game dynamics and facultative cheating in yeast. Nature 459, 253-256, doi:10.1038/nature07921 (2009).

44

45

Zomorrodi, A. R. \& Maranas, C. D. Improving the iMM904 S. cerevisiae metabolic model using essentiality and synthetic lethality data. BMC Syst Biol 4, 178, doi:10.1186/1752-0509-4-178 (2010). 
28 Orth, J. D. et al. A comprehensive genome-scale reconstruction of Escherichia coli metabolism--2011. Mol Syst Biol 7, 535, doi:10.1038/msb.2011.65 (2011).

29 D'Souza, G. \& Kost, C. Experimental Evolution of Metabolic Dependency in Bacteria. PLoS Genet 12, e1006364, doi:10.1371/journal.pgen.1006364 (2016).

30 D'Souza, G., Waschina, S., Kaleta, C. \& Kost, C. Plasticity and epistasis strongly affect bacterial fitness after losing multiple metabolic genes. Evolution 69, 1244-1254, doi:10.1111/evo.12640 (2015).

31 Pande, S. et al. Fitness and stability of obligate cross-feeding interactions that emerge upon gene loss in bacteria. ISME J 8, 953-962, doi:10.1038/ismej.2013.211 (2014).

32 Sanchez, A. \& Gore, J. feedback between population and evolutionary dynamics determines the fate of social microbial populations. PLoS Biol 11, e1001547, doi:10.1371/journal.pbio.1001547 (2013).

33 Elena, S. F. \& Lenski, R. E. Evolution experiments with microorganisms: the dynamics and genetic bases of adaptation. Nat Rev Genet 4, 457-469, doi:10.1038/nrg1088 (2003).

34 Orth, J. D., Thiele, I. \& Palsson, B. What is flux balance analysis? Nat Biotechnol 28, 245-248, doi:10.1038/nbt.1614 (2010).

35 Segrè, D., Vitkup, D. \& Church, G. M. Analysis of optimality in natural and perturbed metabolic networks. Proc Natl Acad Sci U S A 99, 15112-15117, doi:10.1073/pnas.232349399 (2002).

36 Stolyar, S. et al. Metabolic modeling of a mutualistic microbial community. Mol Syst Biol 3, 92, doi:10.1038/msb4100131 (2007).

37 Zomorrodi, A. R. \& Maranas, C. D. OptCom: a multi-level optimization framework for the metabolic modeling and analysis of microbial communities. PLoS Comput Biol 8, e1002363, doi:10.1371/journal.pcbi.1002363 (2012).

38 Zhuang, K. et al. Genome-scale dynamic modeling of the competition between Rhodoferax and Geobacter in anoxic subsurface environments. ISME J 5, 305316, doi:10.1038/ismej.2010.117 (2011).

39 Cressman, R. \& Tao, Y. The replicator equation and other game dynamics. Proc Natl Acad Sci $U$ S A 111 Suppl 3, 10810-10817, doi:10.1073/pnas.1400823111 (2014).

40 Post, D. M. \& Palkovacs, E. P. Eco-evolutionary feedbacks in community and ecosystem ecology: interactions between the ecological theatre and the evolutionary play. Philos Trans R Soc Lond B Biol Sci 364, 1629-1640, doi:10.1098/rstb.2009.0012 (2009).

41 Rauch, J., Kondev, J. \& Sanchez, A. Cooperators trade off ecological resilience and evolutionary stability in public goods games. $J R$ Soc Interface 14, doi:10.1098/rsif.2016.0967 (2017).

42 Widder, S. et al. Challenges in microbial ecology: building predictive understanding of community function and dynamics. ISME J 10, 2557-2568, doi:10.1038/ismej.2016.45 (2016). 
143 Paczia, N. et al. Extensive exometabolome analysis reveals extended overflow 2 metabolism in various microorganisms. Microb Cell Fact 11, 122, 3 doi:10.1186/1475-2859-11-122 (2012).

444 Valle, J. et al. The amino acid valine is secreted in continuous-flow bacterial biofilms. J Bacteriol 190, 264-274, doi:10.1128/JB.01405-07 (2008).

\section{Figures' Legends}

9 Figure 1. Outline of the proposed genome-driven evolutionary game theory

10 approach. Annotated genomes of community members are used to construct 11 genome-scale metabolic models. For each possible pair of genotypes in the

12 community, constraint-based analysis tools for metabolic models such as Flux

13 Balance Analysis ${ }^{34}$ are used to estimate the fitness (or "payoff") of each genotype as

14 they engage in a specific metabolite-mediated interaction. These payoffs form the

15 payoff matrix of the game. Based on this payoff matrix we identify all Nash equilibria 16 of the game, using a newly developed automated tool (NashEq Finder, see

17 supplementary text). The payoff matrix also allows us to model evolutionary 18 dynamics (i.e., how genotype frequencies change over time) ${ }^{25}$ and to determine 19 which of the identified Nash equilibria are evolutionarily stable (see Methods).

21 Figure 2. Metabolic dependencies in populations of $\boldsymbol{S}$. cerevisiae growing on 22 sucrose and in populations of $E$. coli secreting an amino acid. (A) Metabolic 23 interactions between producer (wild-type, WT) and non-producer (mutant, MT) 24 genotypes of $S$. cerevisiae growing on sucrose ${ }^{26}$. Here, $e$ represetns the percentage 25 of glucose/fructose that diffuses away and serves as a public good. (B) Nash 26 equilibria and $(\mathrm{C})$ the equilibrium frequency of WT for the community shown in (A) 27 as a function of the capture efficiency of the glucose/fructose and the invertase 28 production cost (modeled by changing the stoichiometric coefficient of ATP in the 29 sucrose hydrolysis reaction, indicated as $x$ ). An alternative in silico formulation of 30 the energetic cost of invertase production that reproduces exactly the setup used in 31 the experiment by Gore et al ${ }^{26}$ (based on histidine auxotrophy) proved to be 
1 qualitatively equivalent to the analysis presented here (see supplementary text for 2 details). The equilibrium frequency of WT was obtained from in silico invasion

3 experiments (see Methods) for two cases of a small fraction of MT invading a 4 resident population of WT and vice versa demonstrated that the equilibrium 5 frequency of WT is the same in both cases. (D) Metabolic interactions between WT 6 and MT when additional glucose is provided in the growth medium (see 7 supplementary text for details). (E) Nash equilibria and (F) the equilibrium

8 frequency of WT in the presence of glucose in the growth medium. The entire

9 Snowdrift game region and part of the Mutually Beneficial region in (B) are replaced

10 by the Prisoner's Dilemma game, in (E), which is consistent with previous reports 26

11 and serves as an additional verification of our modeling approach. This is because in

12 the presence of glucose, MT is less dependent on WT thereby increasing the average

13 fitness of MT. (G) Possible genotypes in populations of E. coli leaking an amino acid

14 include a prototrophic wild-type strain (WT) self-synthesizing a leaky amino acid

15 and a mutant strain (MT) lacking the gene(s) for the biosynthesis of this amino acid.

16 (H) The identified Nash equilibria for various leakiness levels (as a percentage of an

17 in silico determined maximum: see supplementary text) across all 20 amino acids.

18 Amino acids are shown here by using their standard three-letter code in the order of

19 increasing in silico growth cost (see also supplementary Figure S1). (I) The

20 equilibrium frequency of WT as a function of the leakiness level and amino acid

21 type. In silico invasion experiments for two cases of MT invading WT and WT

22 invading MT revealed that the equilibrium frequencies are insensitive to the initial

23 frequencies. (J) Experimentally reported leakiness levels of amino acids averaged

24 over three different datasets 43,44 . Values in each dataset were normalized to their

25 maximum (see supplementary Table S1 for values of data). Error bars show

26 standard deviation over the three datasets. (K) Predicted selection coefficients

27 across all amino acids and leakiness levels vs. the experimentally reported ones for

28 E. coli ${ }^{29}$. Empty circles show the average predicted selection coefficient for each

29 amino acid. The black and blue lines show the fitted lines to the predicted and

30 experimental values ${ }^{29}$, respectively. The equation for the fitted line to experimental

31 data was inferred from a graph of reference ${ }^{29}$. 
2 Figure 3. Equilibrium metabolic dependencies in populations of $E$. coli with

3 two leaky amino acids. (A) Genotypes involved include a prototrophic strain self-

4 synthesizing two leaky amino acids (i.e., 11), two single-mutant strains each lacking

5 the gene(s) for the biosynthesis of one amino acid but synthesizing and leaking the

6 other (i.e., 01 and 10), and a mutant strain lacking the genes for the biosynthesis of

7 both leaky amino acids (i.e., 00). Here, ' 1 ' and '0' denote the presence or absence of

8 biosynthesis pathways (genes) for an amino acid, respectively. (B) The identified

9 Nash equilibria of two-player games (i.e., pairwise interactions) for all amino acids

10 pair across different leakiness levels, zoomed in for four selected pairs including (C)

11 (lysine, isoleucine), (D) (glutamate and leucine), (E) (arginine, glutamate) and (F)

12 (glycine, threonine). A sample payoff matrix of the game is shown in (C) for a

13 leakiness level of $15 \%$ for both lysine and isoleucine. Non-viable equilibria signify

14 associations between genotypes that are non-viable leading to community collapse.

15 Nash equilibria of three- and four-player games for a selected number of amino acid

16 pairs are also given in supplementary Figures S2-S7. Metabolic maps in (E) And (F)

17 show the metabolic pathways involved in the synthesis of the respective amino

18 acids. Sample payoff matrices in the region of sustainable leakiness levels in (E) and

19 (F) are given in supplementary Figure S8.

21 Figure 4. Impact of the initial genotype frequencies on the evolutionary 22 emergence of metabolic dependencies in populations of $E$. coli with two leaky

23 amino acids. Here, we have shown the results of targeted in silico invasion 24 experiments for a representative amino acid pair (lysine, isoleucine) (see also 25 Figure 3c). (A) 00, 01 and 10 simultaneously originate from 11 (i.e., wild-type) 26 through genome streamlining and invade an existing population of 11 genotypes. 27 (B) A small population of 10 and 00 invade a resident population of the 11 and 01. 28 This simulates the second step of a two-step process for the loss of the leaky 29 functions hypothesized in ${ }^{14}$ (see the main text and also supplementary Figure S9). 30 (C) A small population of 01 and 10 invades a resident population of 11 . This models 31 an alternative scenario for the two-step loss of leaky functions leading to stable 
1 cross-feeders: Two partial producer mutant genotypes (01 and 10) originate from

211 , followed by the rise of the 00 genotype from 01 and/or 10. As shown here, 3 cross-feeders can evolutionarily stabilize and co-exist with 11 genotypes in the first

4 step. Further analysis showed that cross-feeders are also resistant to invasion by 00

5 genotypes arising in the second step (see supplementary Figure S10). Dynamic plots

6 in (A)-(C) show the sample evolutionary dynamics of the system for selected equal

7 leakiness levels for both lysine and isoleucine. Pie charts show the equilibrium

8 frequencies of each genotype starting from the initial genotype frequencies shown

9 in each panel. These equilibrium frequencies are given only for the sustainable

10 leakiness region (green region in Figure 3C). 


\section{Figure 1}

Annotated genomes of target organisms

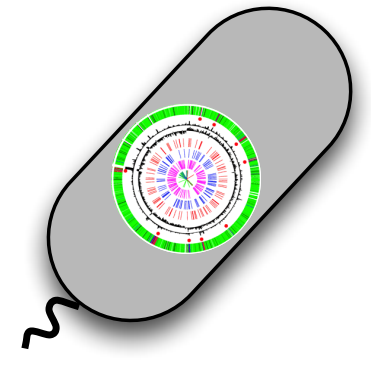

Producer

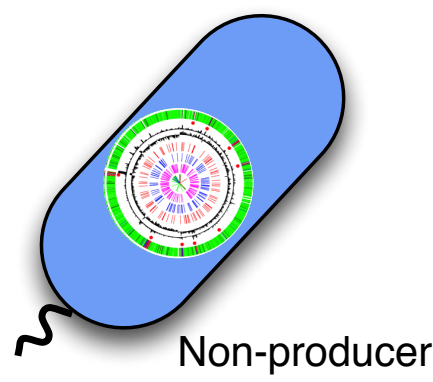

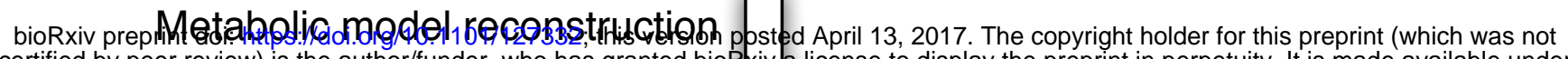
certified by peer review) is the author/funder, who has granted biofiviv license to display the preprint in perpetuity. It is made available under
aCC-BY 4.0 ternational license.

\section{Genome-scale metabolic models}
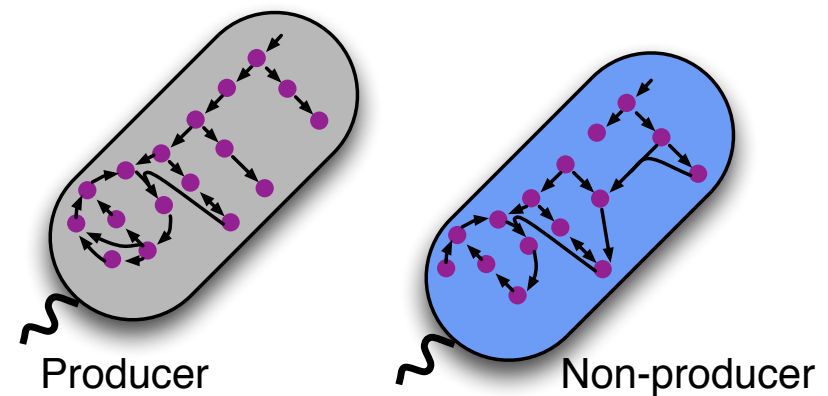

Calculate payoffs using constraint-based methods in all possible pairwise interactions

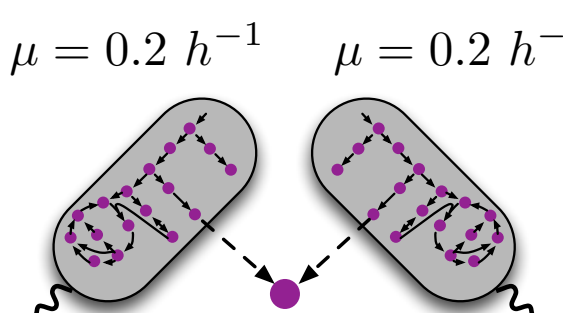

Producer

Producer

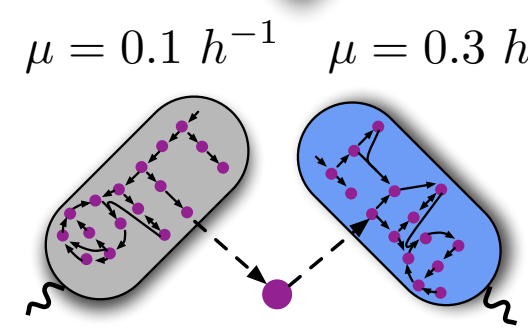

Producer

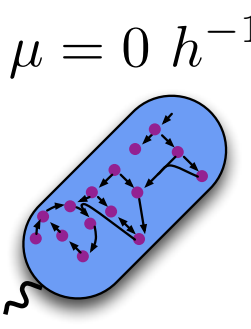

Non-producer Non-producer
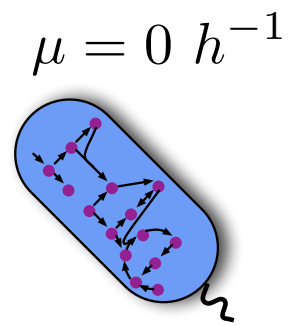

Non-producer

Payoff matrix

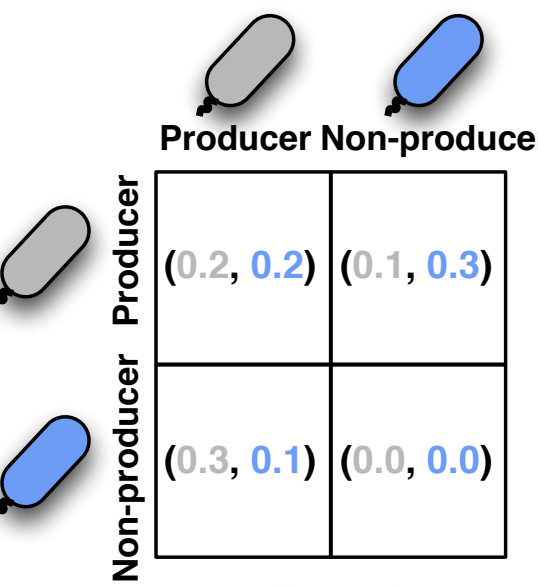

Automated identification of Nash equilibria using NashEq Finder

\section{Evolutionary dynamics} using Replicator Equation<smiles>C1CCCCCC1</smiles>

Nash equilibria

Producer Non-producer

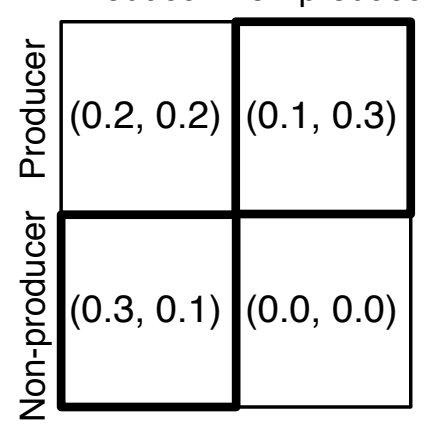

Nash equilibrium $=$ (Producer, Non-producer $)$
Evolutionary dynamics

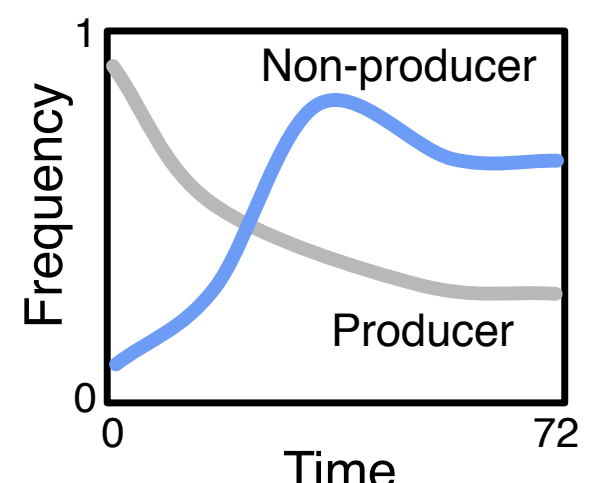


(A)

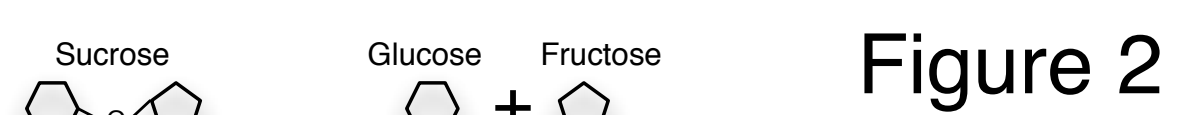

(B)

Wild-type Producer (WT) Mutant Non-producer (MT)

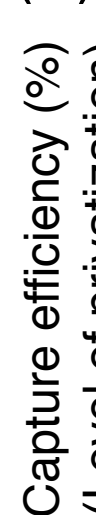

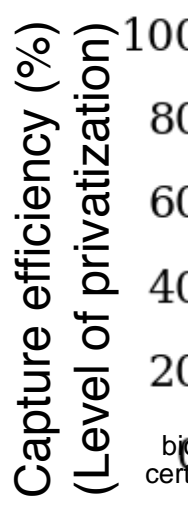

(C) EQUILIBRIUM FREQUENCY OF WT

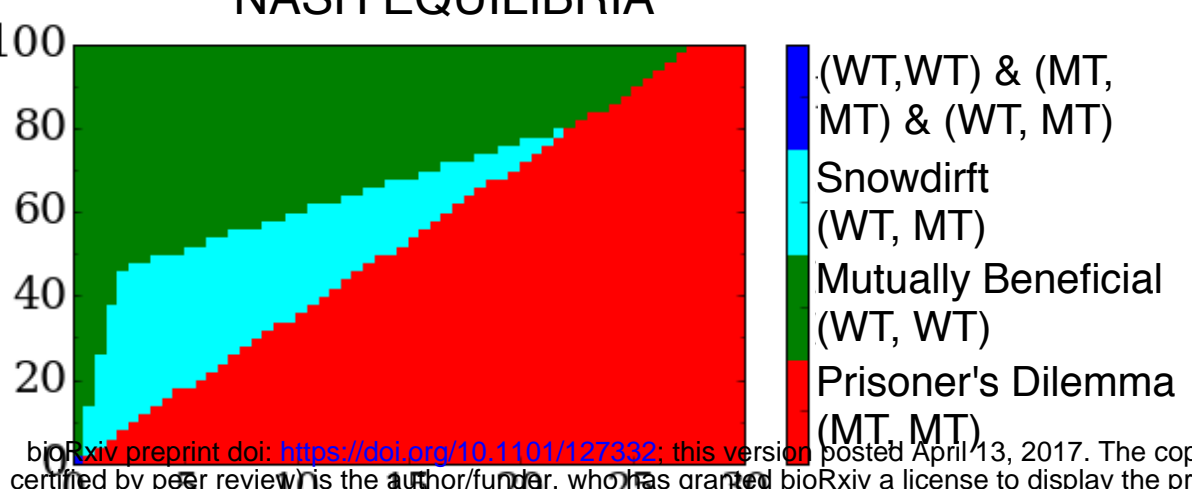

Stoichiometric coefficient of ATP

(Cost of sucrose hydrolysis)

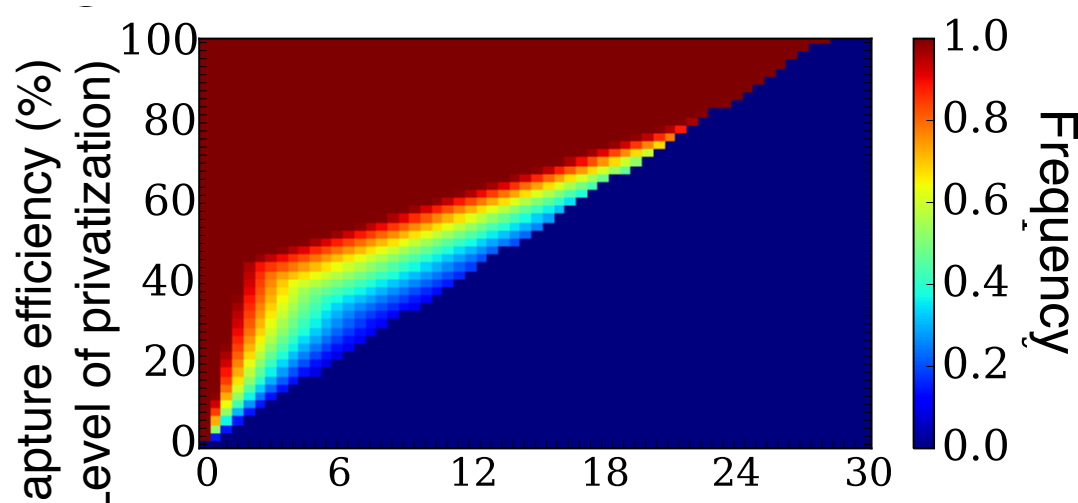

Stoichiometric coefficient of ATP

(Cost of sucrose hydrolysis)

(G)

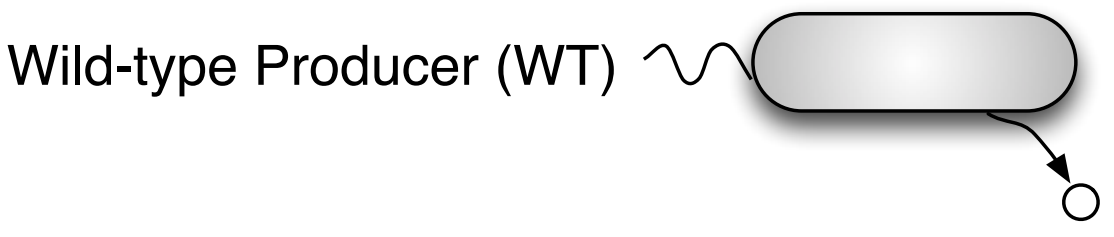

(H)

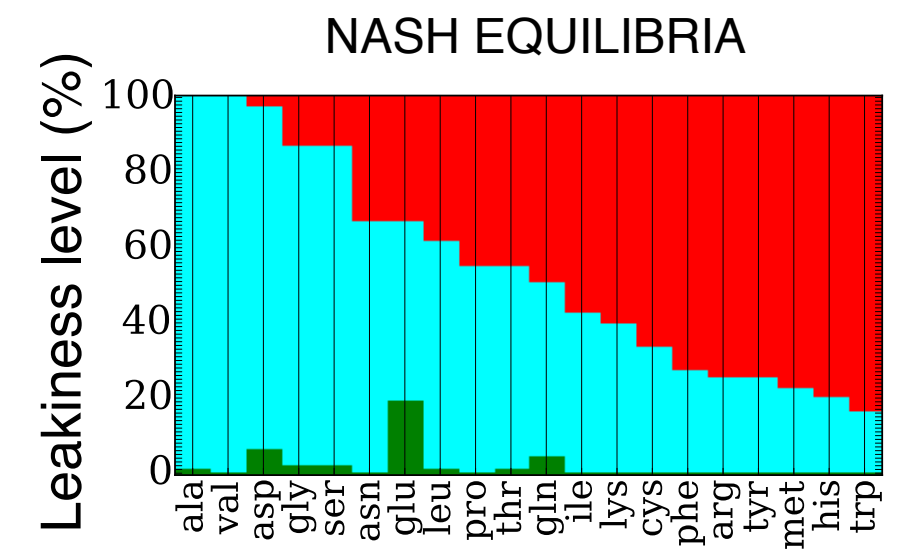

(MT, MT) \&

(MT, WT)

Snowdrift

(MT, WT)

Mutually Beneficial (WT, WT)

Prisoner's Dilemma (MT, MT)

Amino acids (in increasing growth cost)

(I)

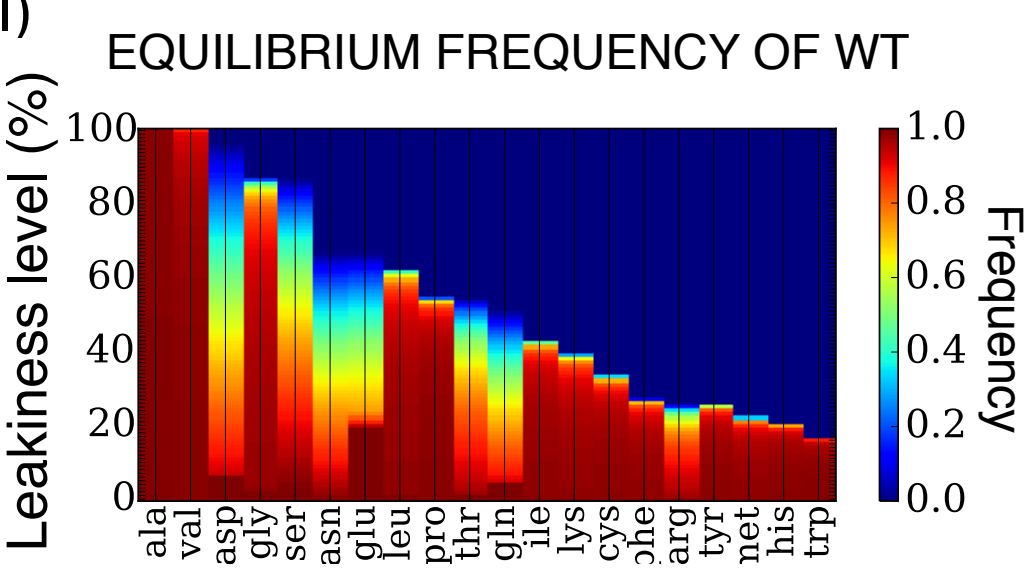

Amino acids (in increasing growth cost)
(D)

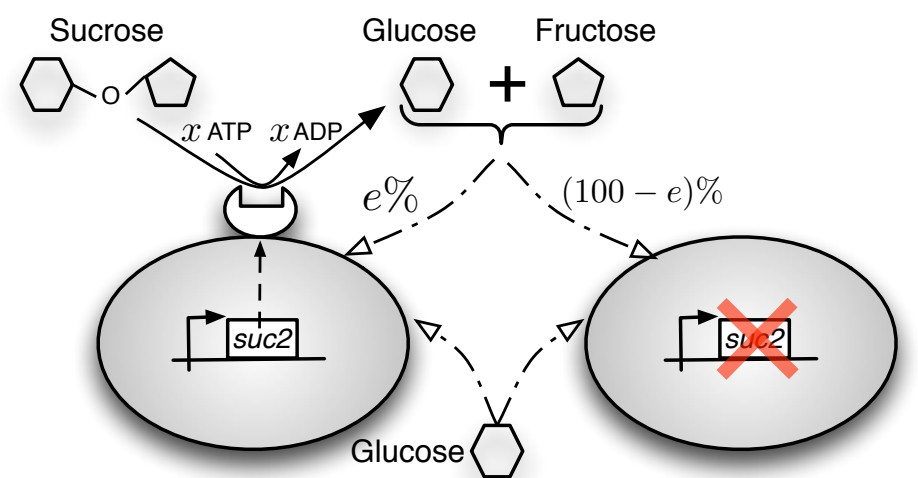

(E)

Wild-type Producer (WT) $\uparrow$ Mutant Non-producer (MT) NASH EQUILIBRIA

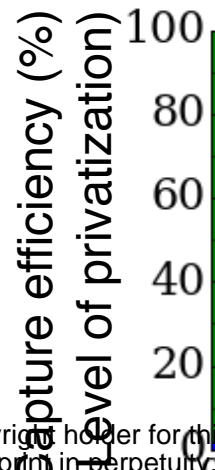$$
0
$$

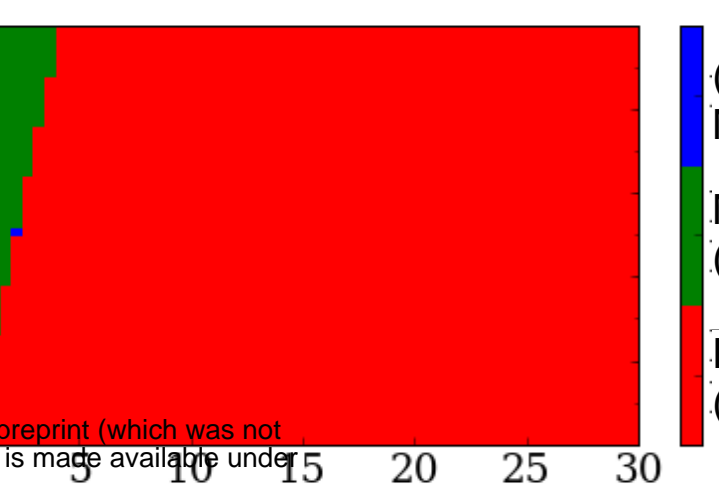

WT, WT) \& (MT, MT) \& (WT, MT)

Mutually Beneficial (WT, WT)

Prisoner's Dilemma (MT, MT)

Stoichiometric coefficient of ATP

(Cost of sucrose hydrolysis)

(F)

EQUILIBRIUM FREQUENCY OF WT
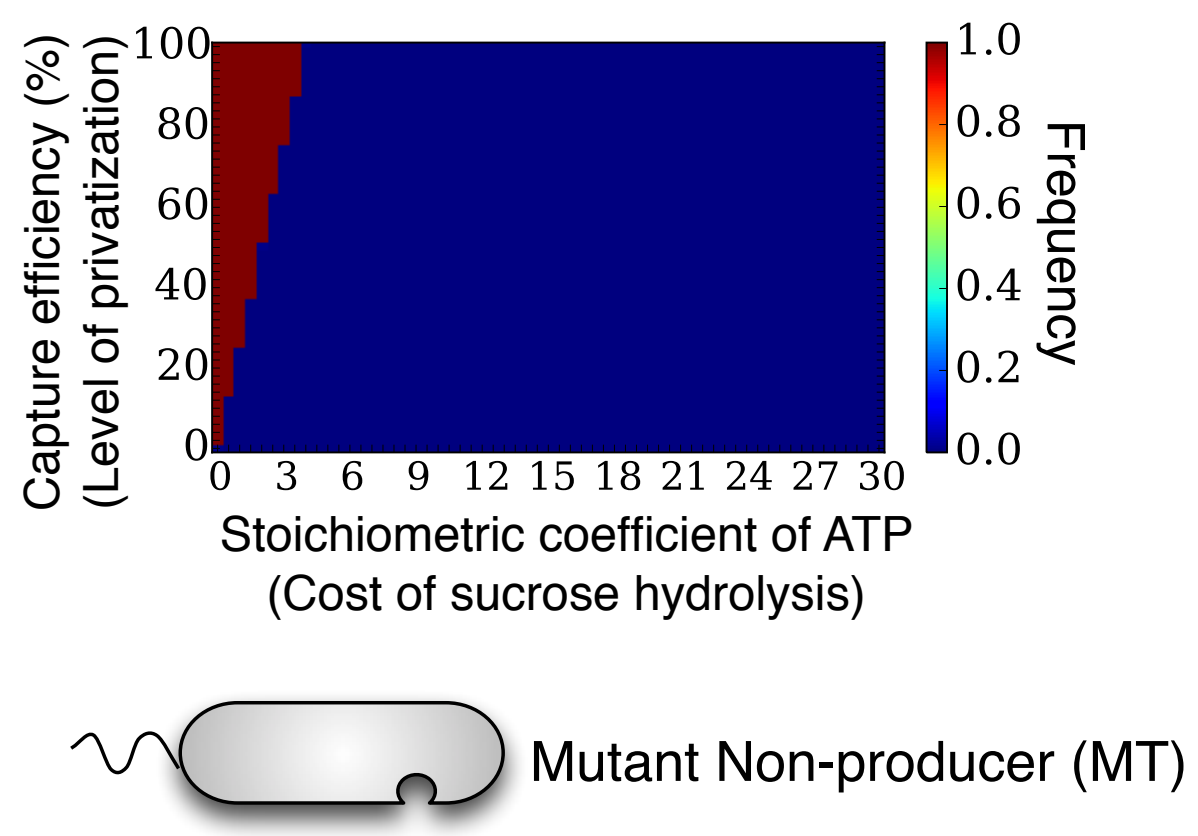

(J) AVERAGE SECRETION LEVEL OF AMINO

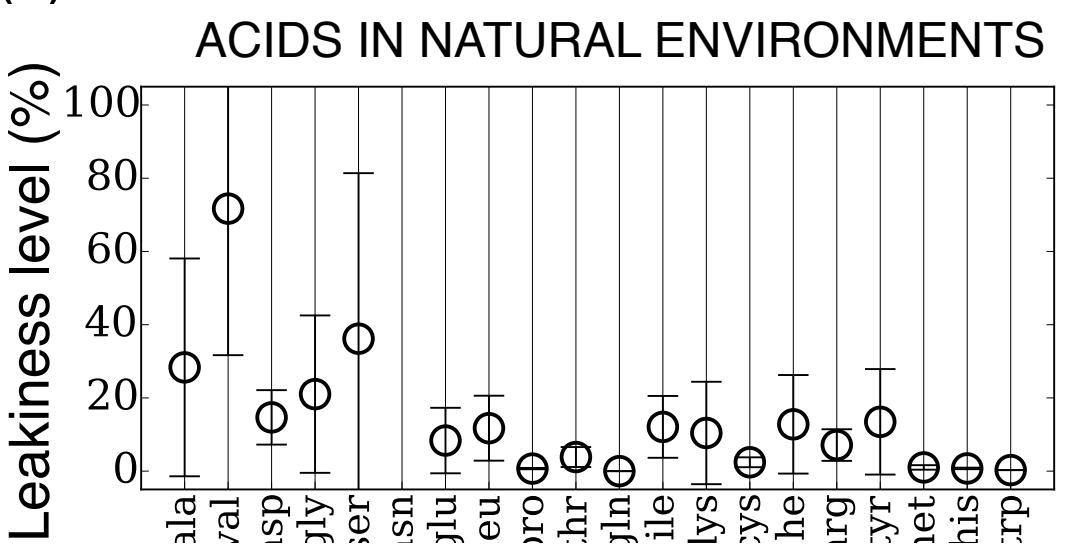

Amino acids (in increasing growth cost)

(K)

PREDICTED VS. MEASURED SELECTION COEFFS

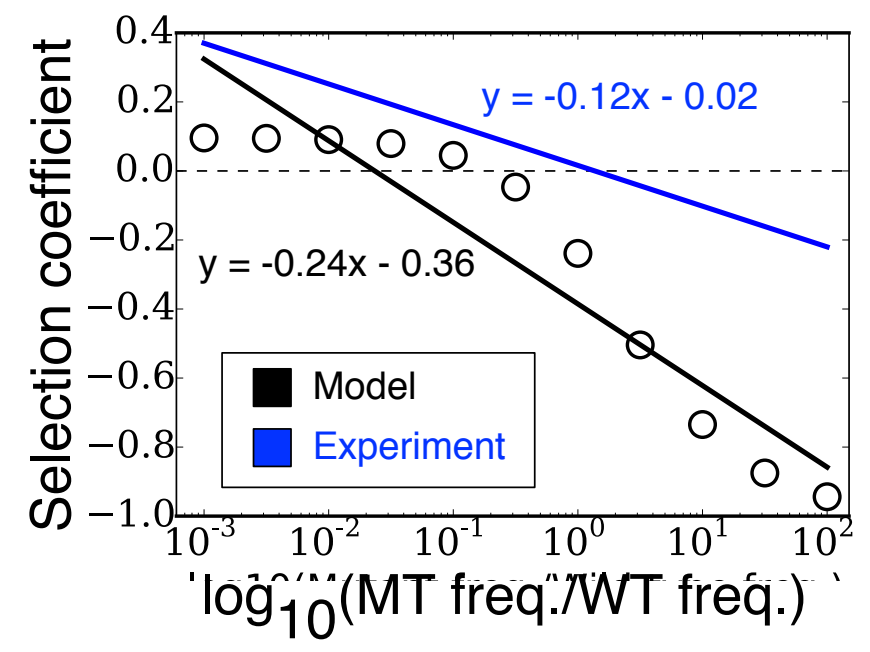


(A)

Figure 3

(B)

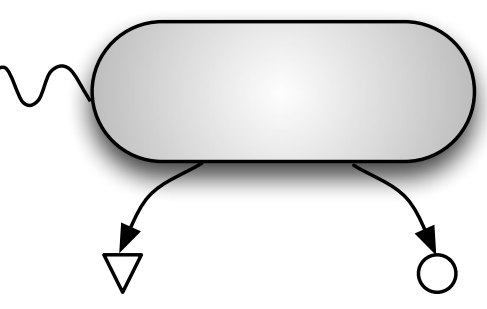

01

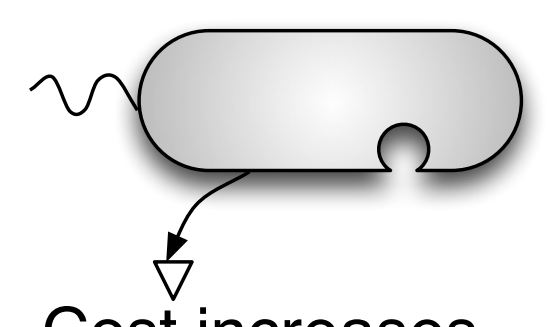

Cost increases

ala val asp gly ser asn glu leu pro thr gln ile lys cys phe arg tyr met his

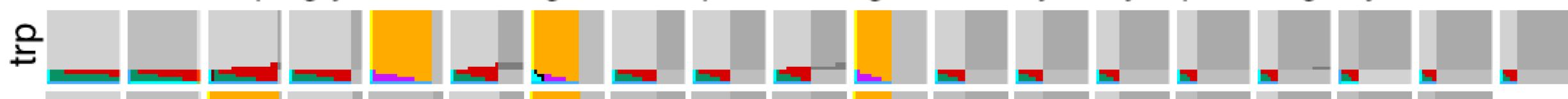

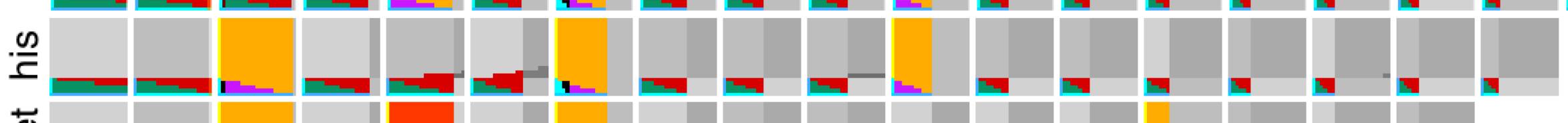

$\stackrel{\square}{\Xi}$

2-1 - -

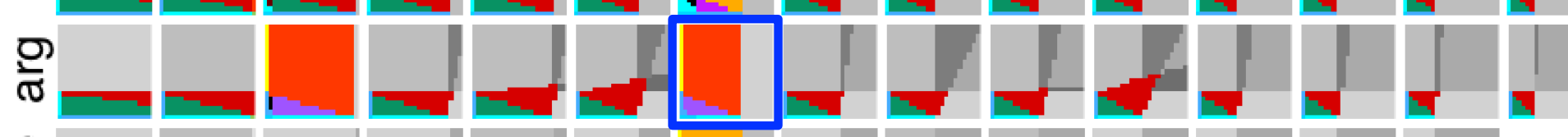
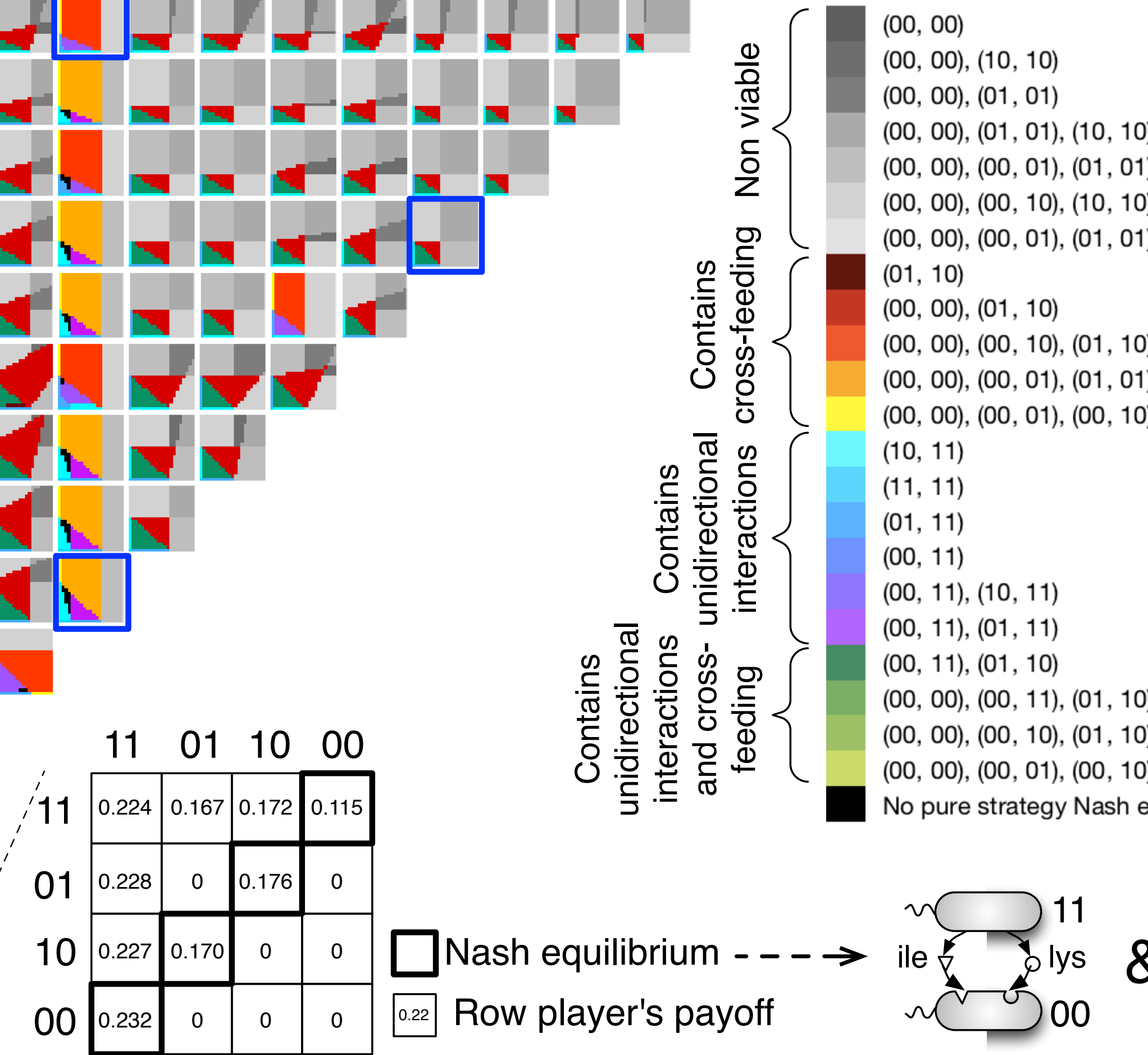

( 20,00$),(01,01),(10,10)$

$\begin{array}{ll}\text { 은 } & (00,00),(00,01),(01,01) \\ (00,00),(00,10),(10,10)\end{array}$

의 $(00,00),(00,01),(01,01),(10,10)$

$(01,10)$

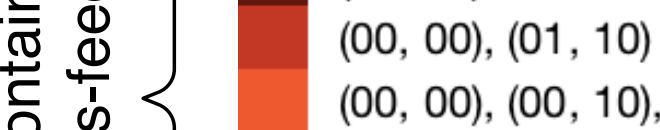

(00, 00), $(00,10),(01,10),(10,10)$

$(00,00),(00,01),(01,01),(01,10)$

$(00,00),(00,01),(00,10),(01,01),(01,10),(10,10)$

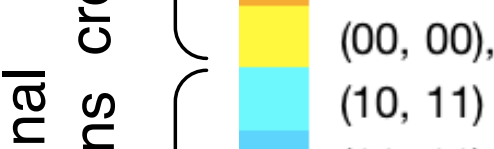
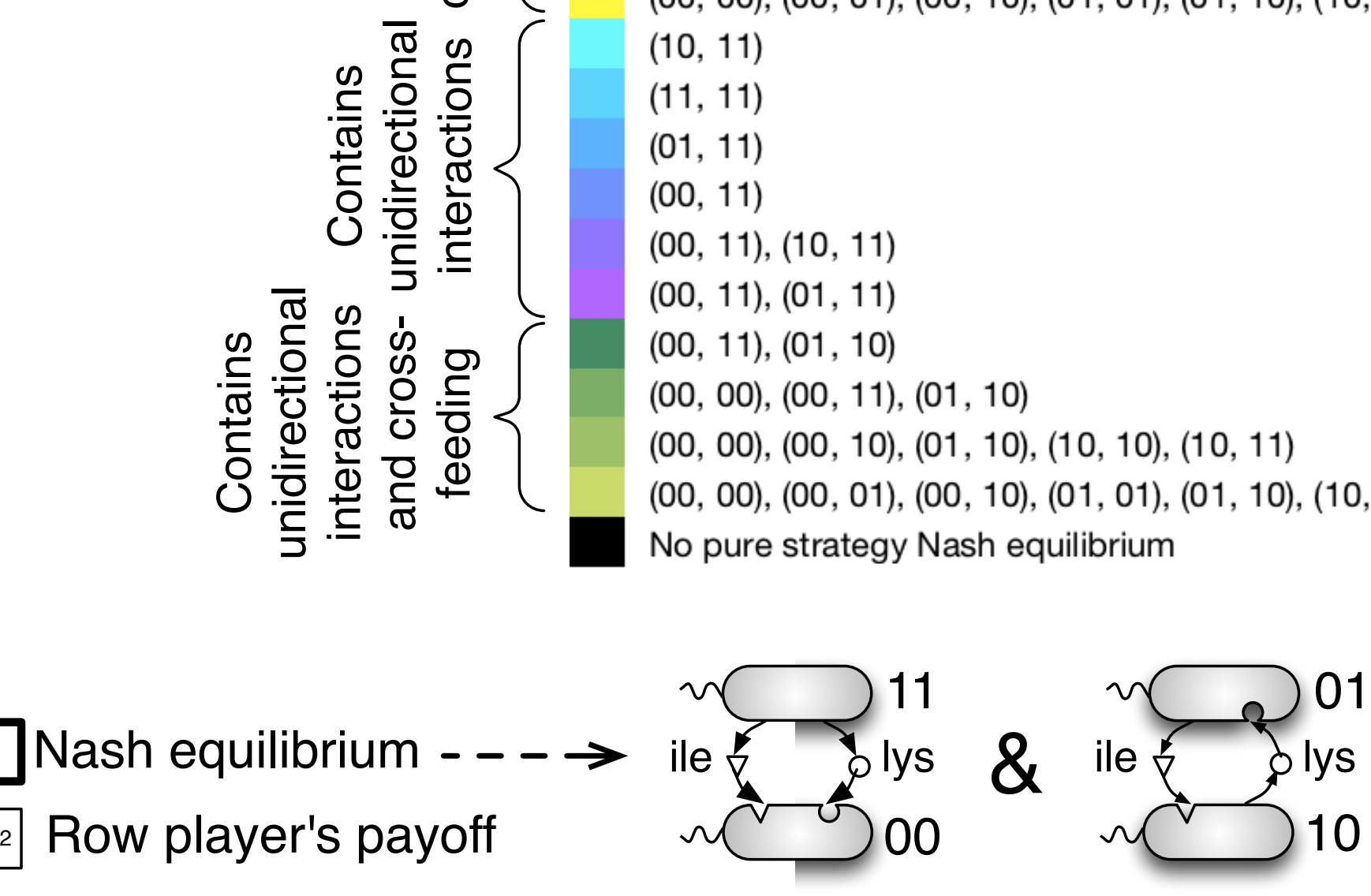

(C)

$(\mathrm{E})$
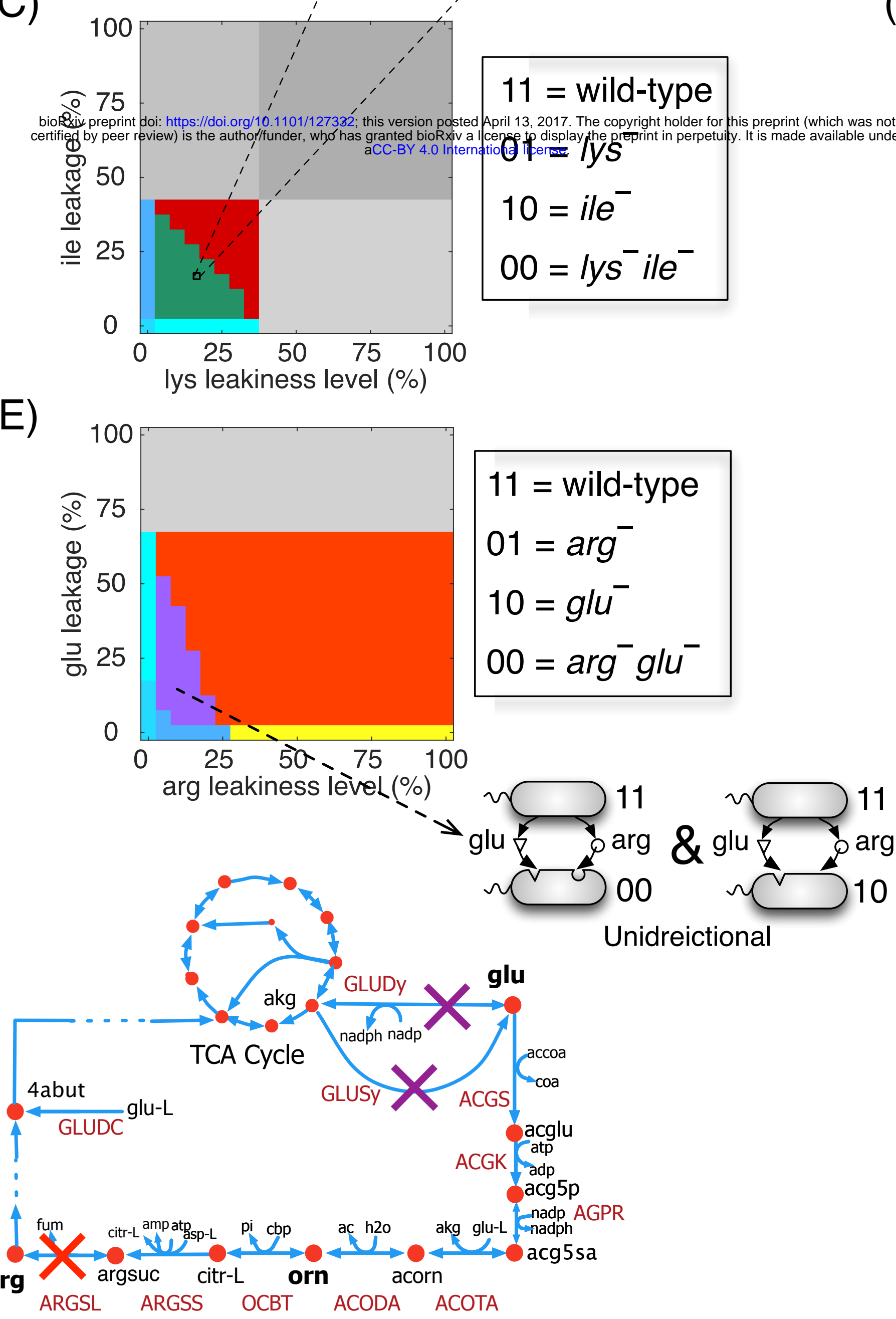

$\mathrm{X}$ Reaction deletions creating glu auxotrophy $\mathrm{X}$ Reaction deletions creating arg auxotrophy
(D)

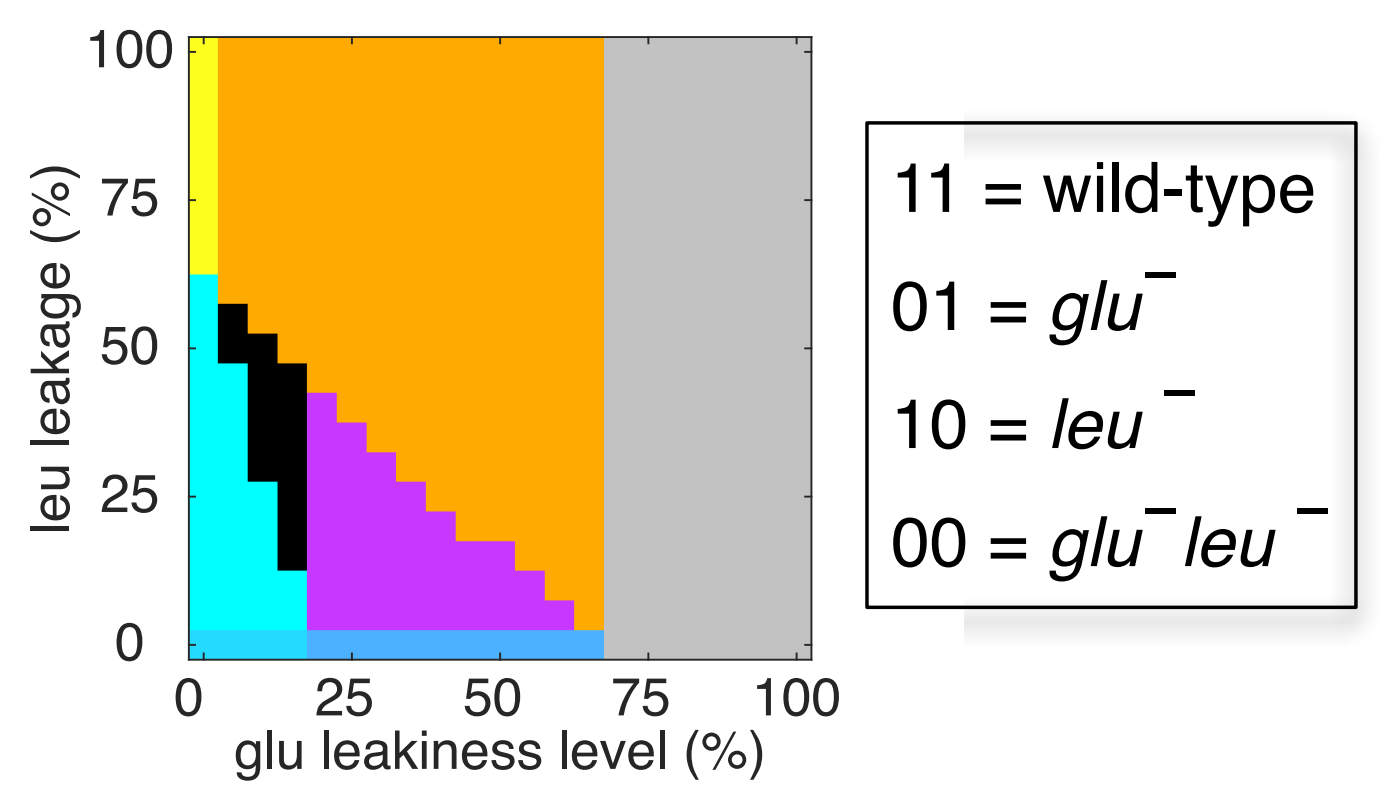

(F)
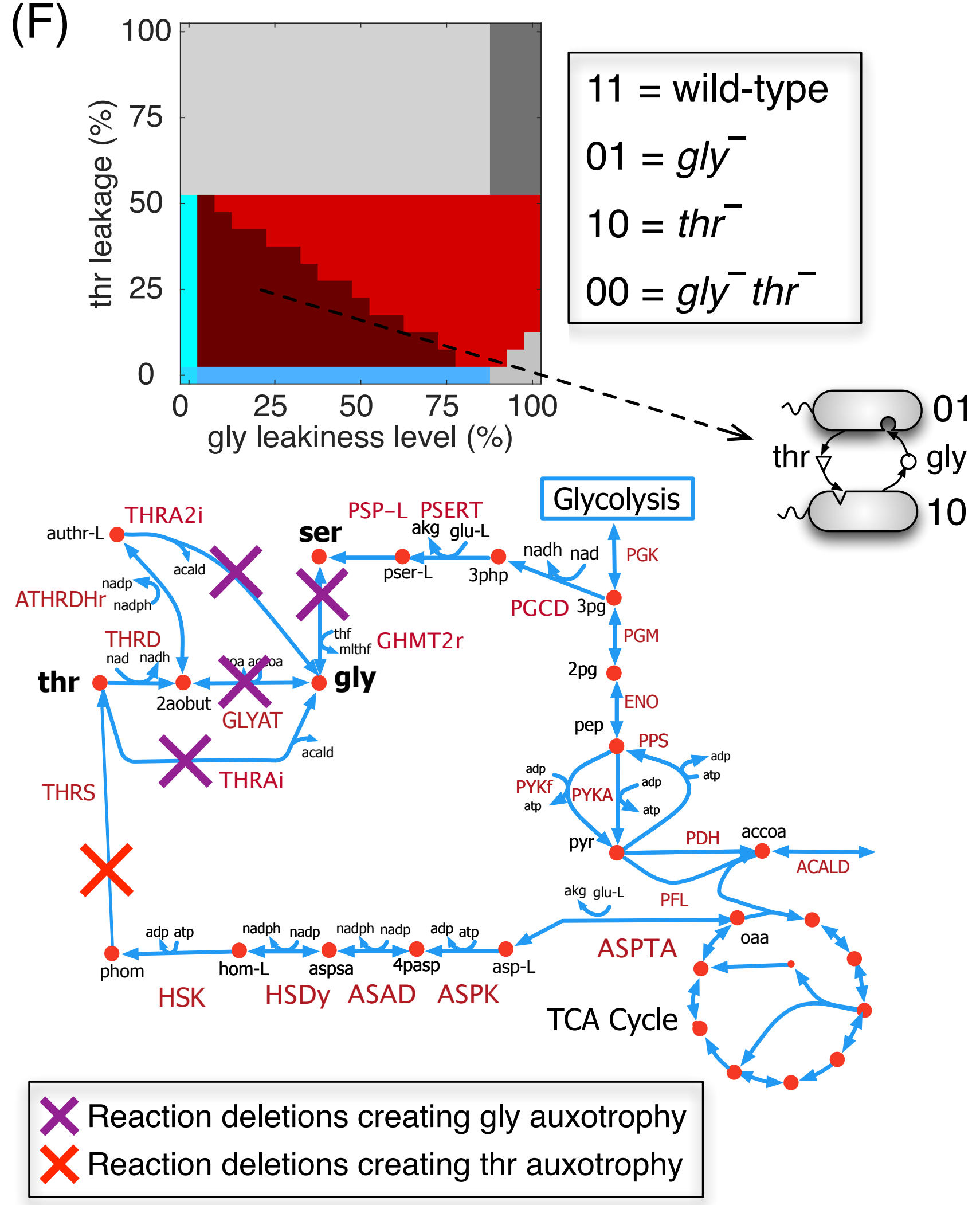
Figure 4

(A)

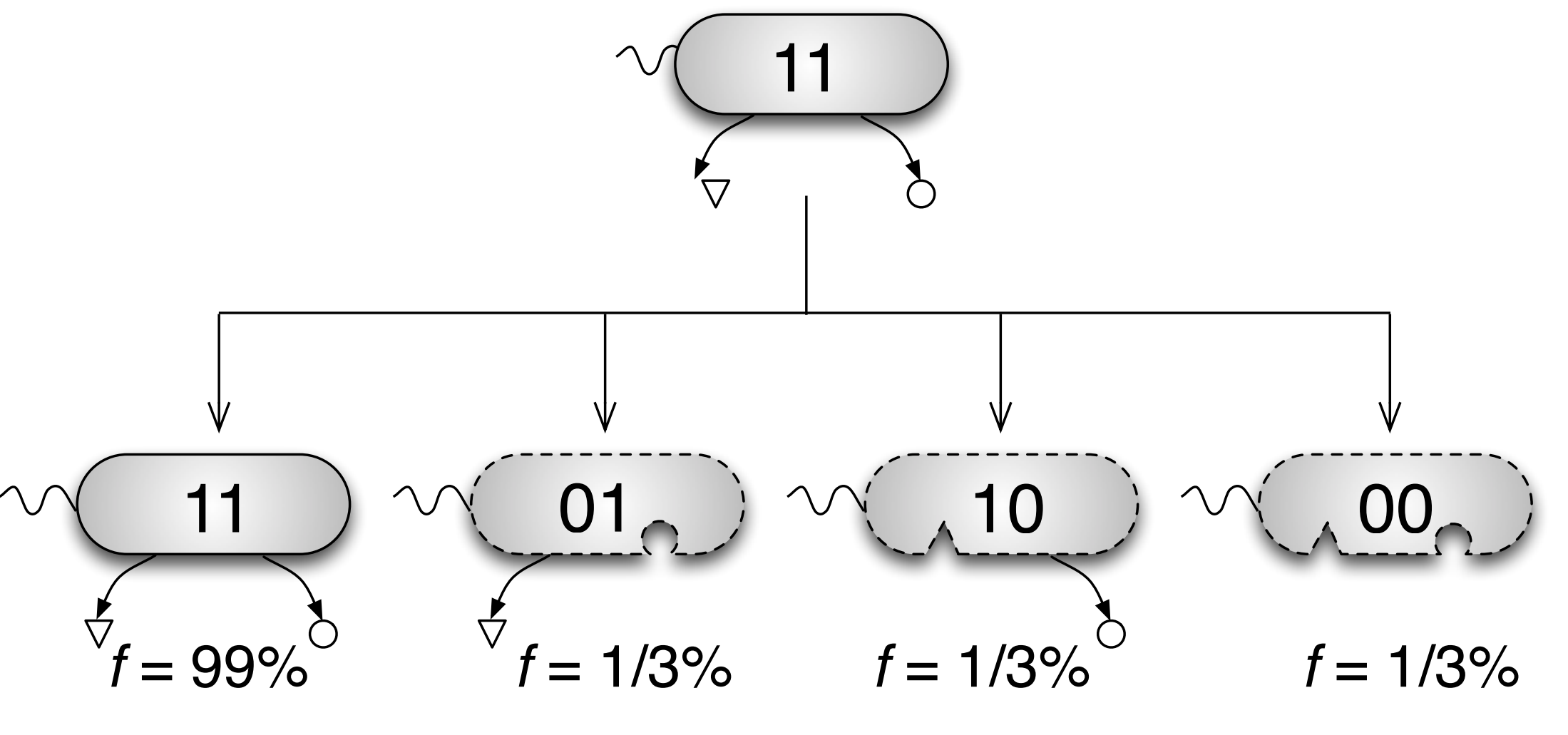

11 (Wild-type $) \quad 01$ (lys $\left.^{-}\right) \quad 10\left(\right.$ ile $\left.^{-}\right) \quad 00\left(\right.$ lys $^{-}$ile $\left.^{-}\right)$

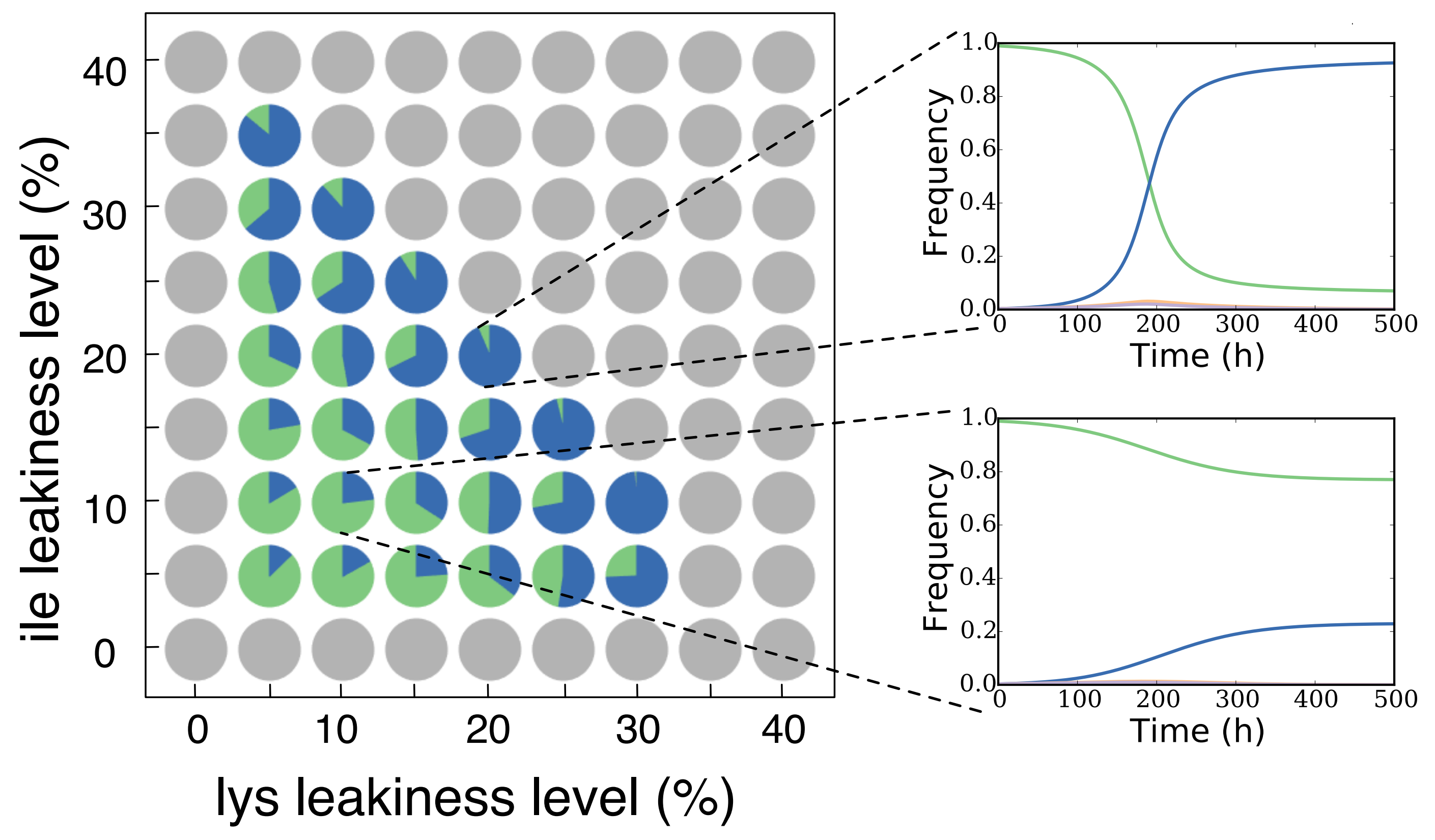

(B)
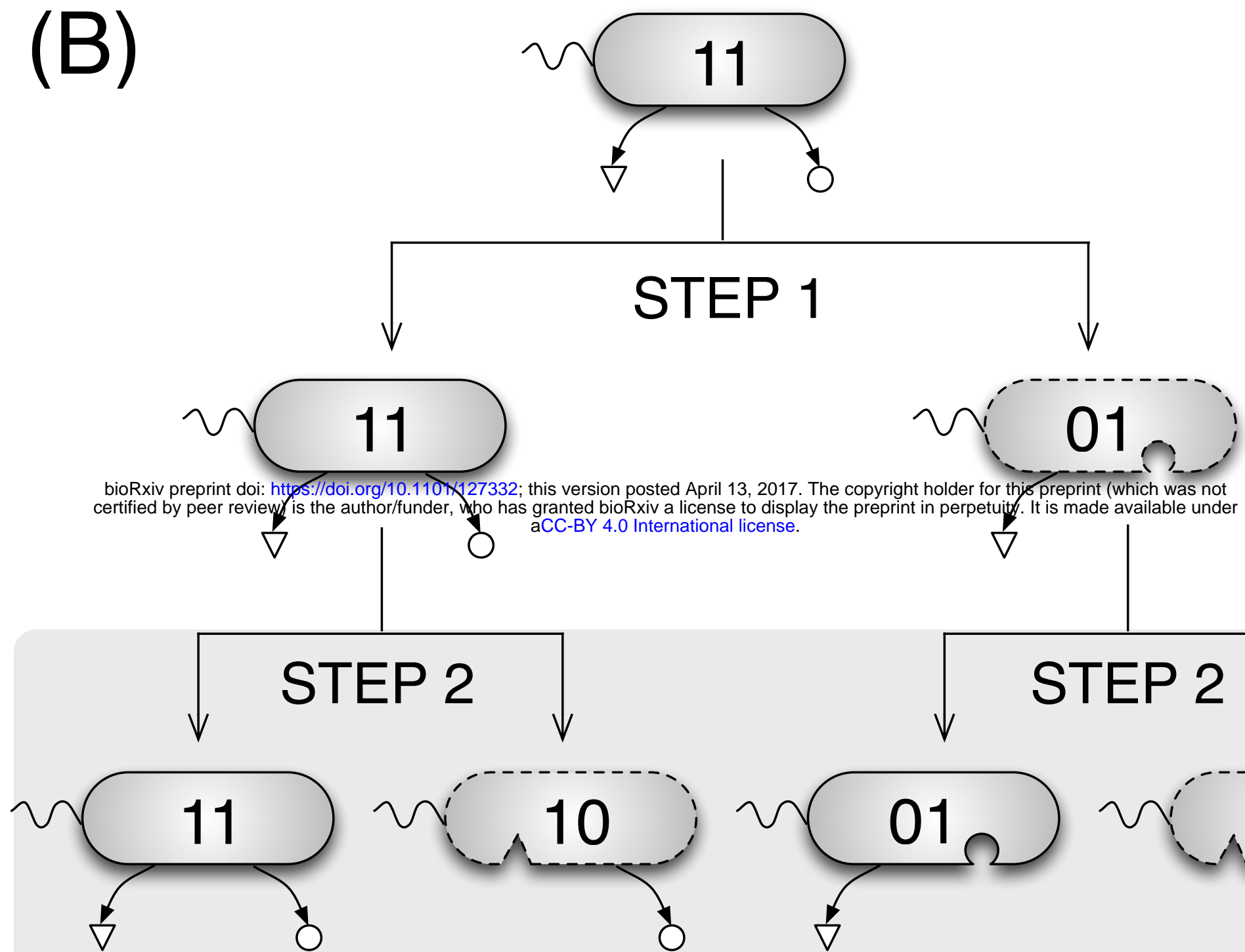

STEP 2

$f=99 / 2 \%$

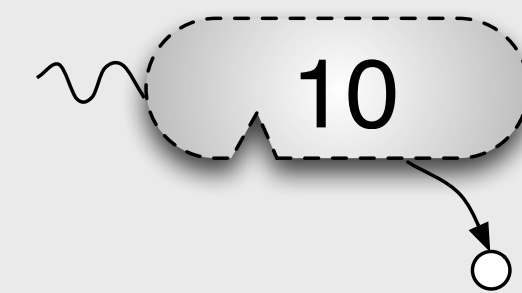

$f=1 / 2 \%$

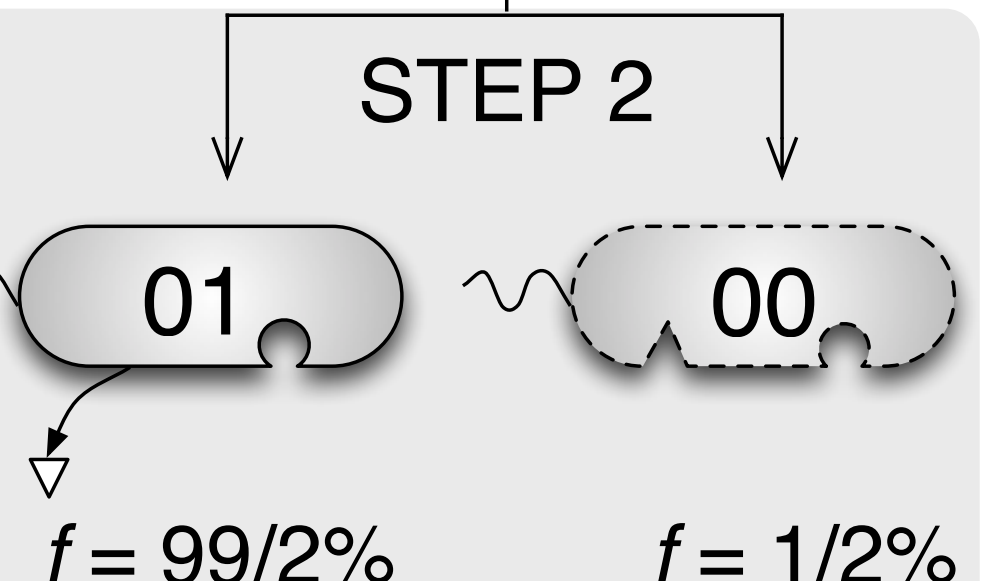

(C)
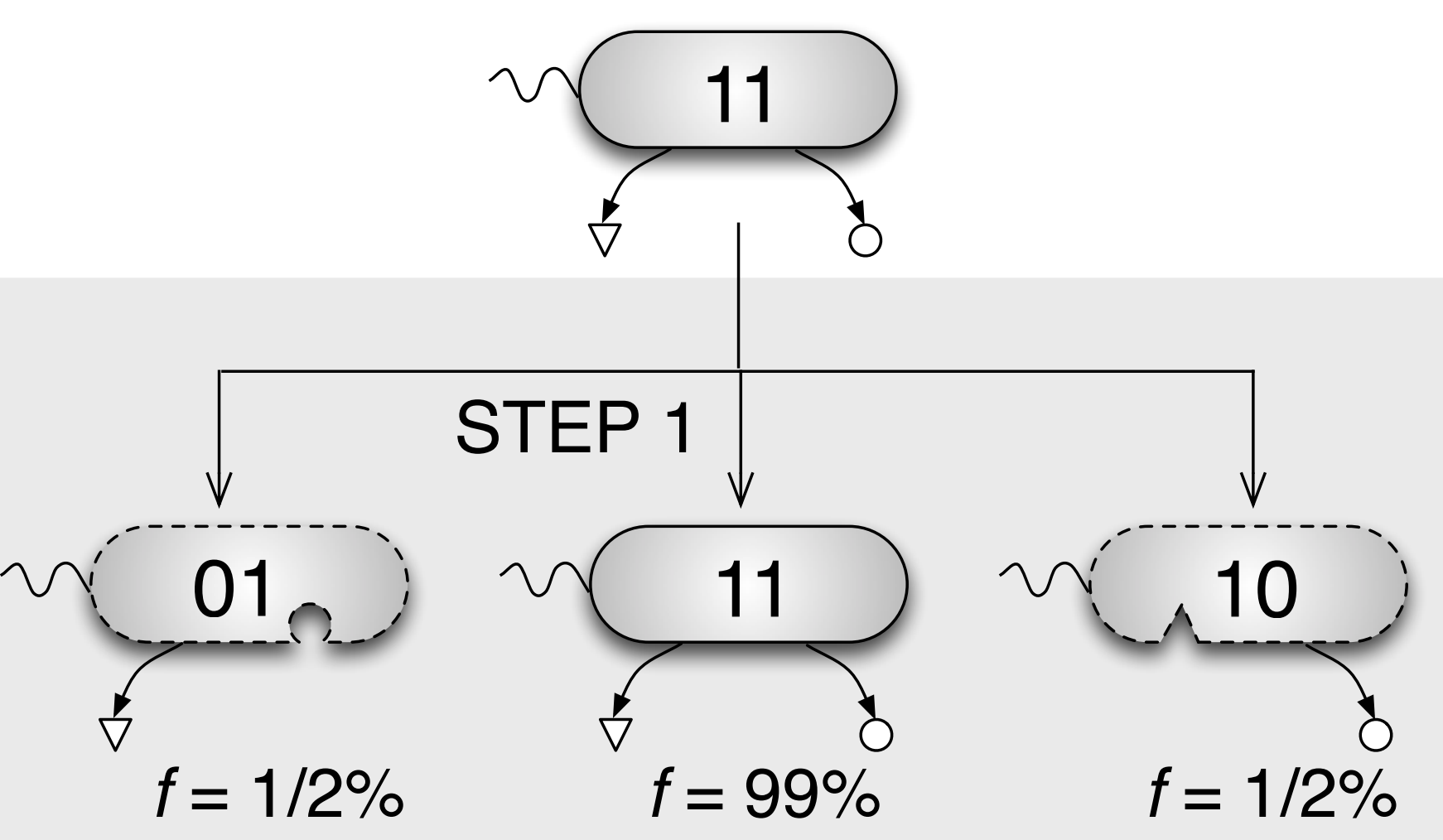

STEP 2

STEP 2

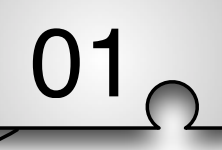

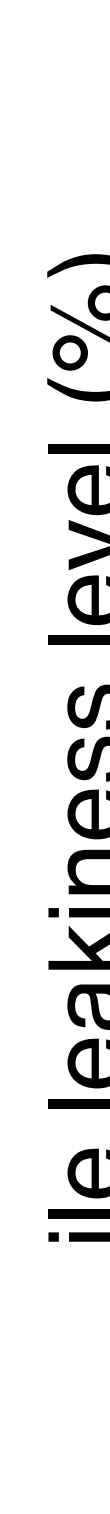
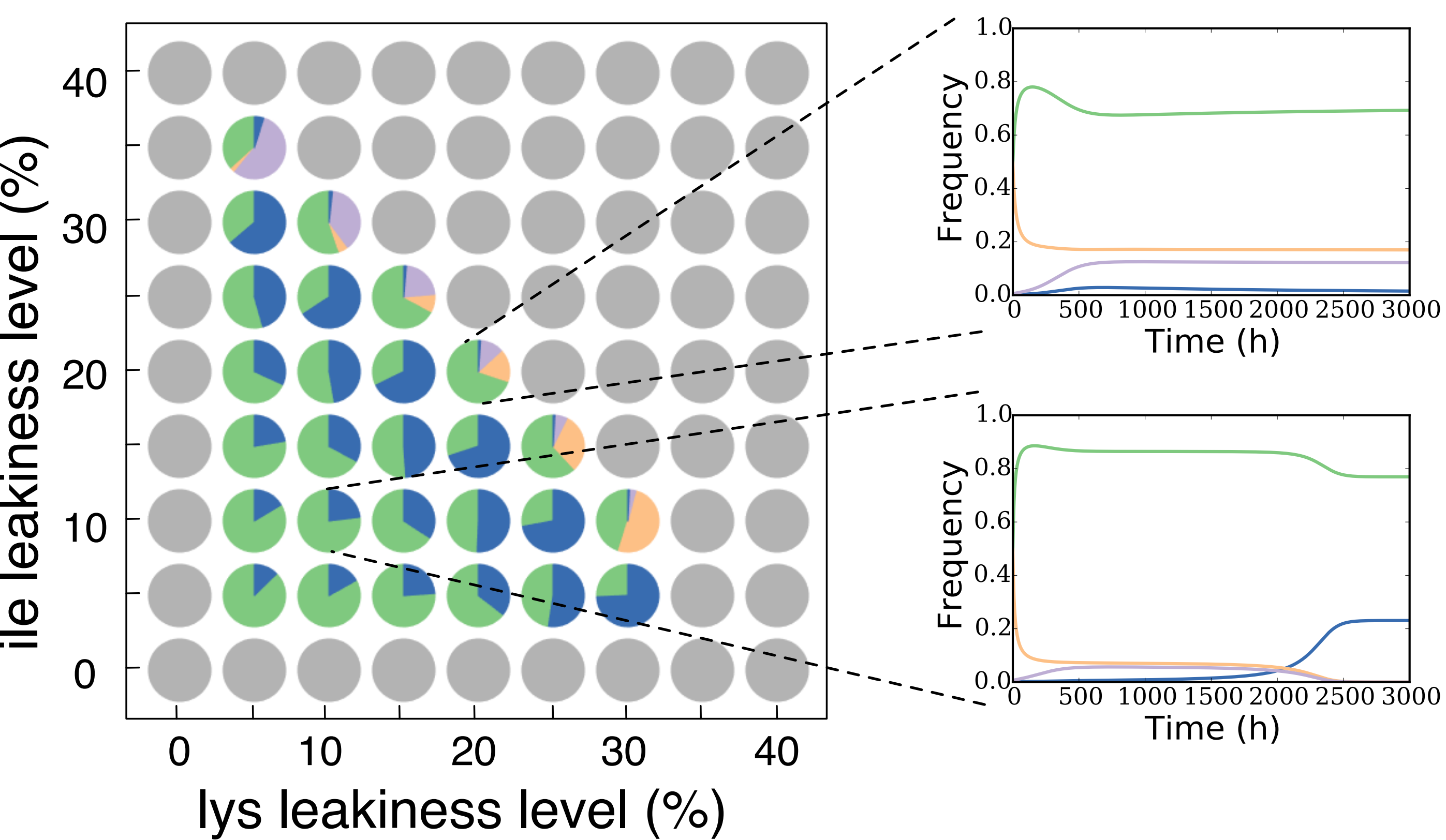

Time (h)

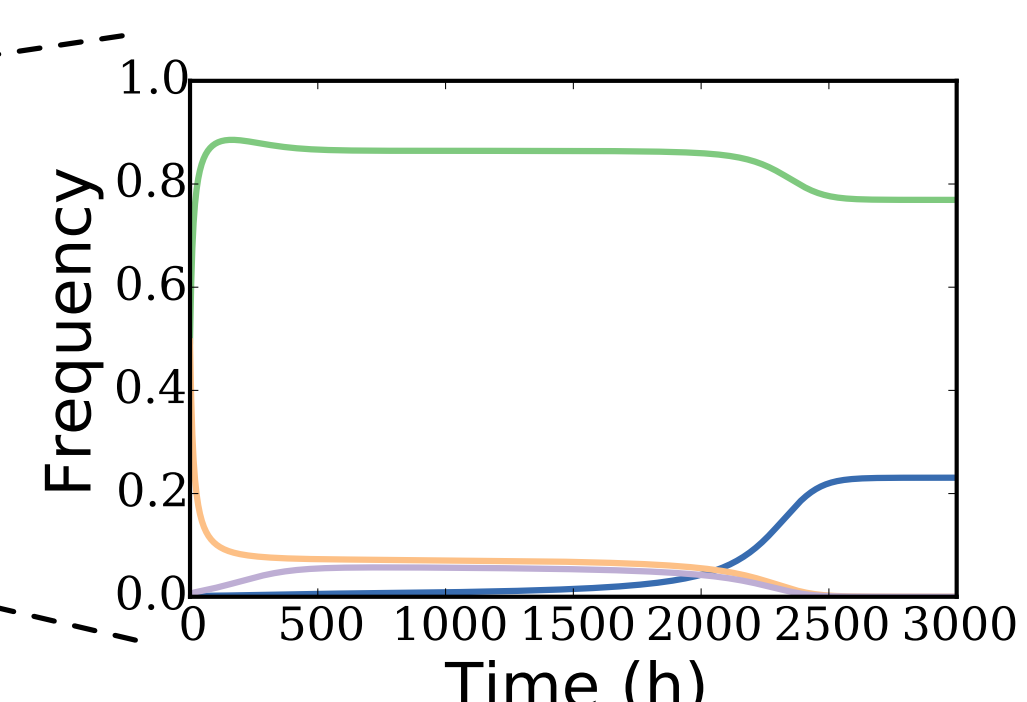

Time $(\mathrm{h})$

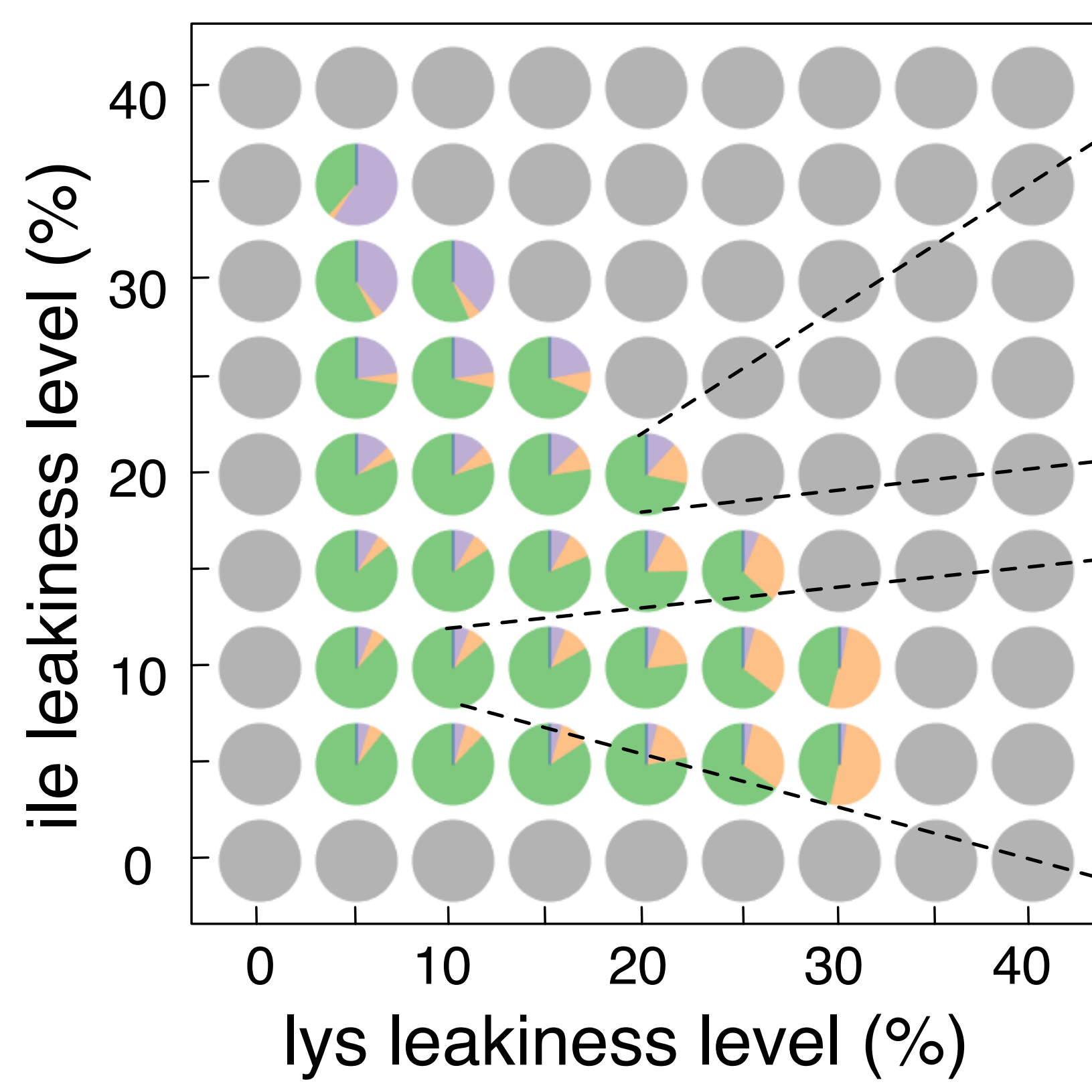

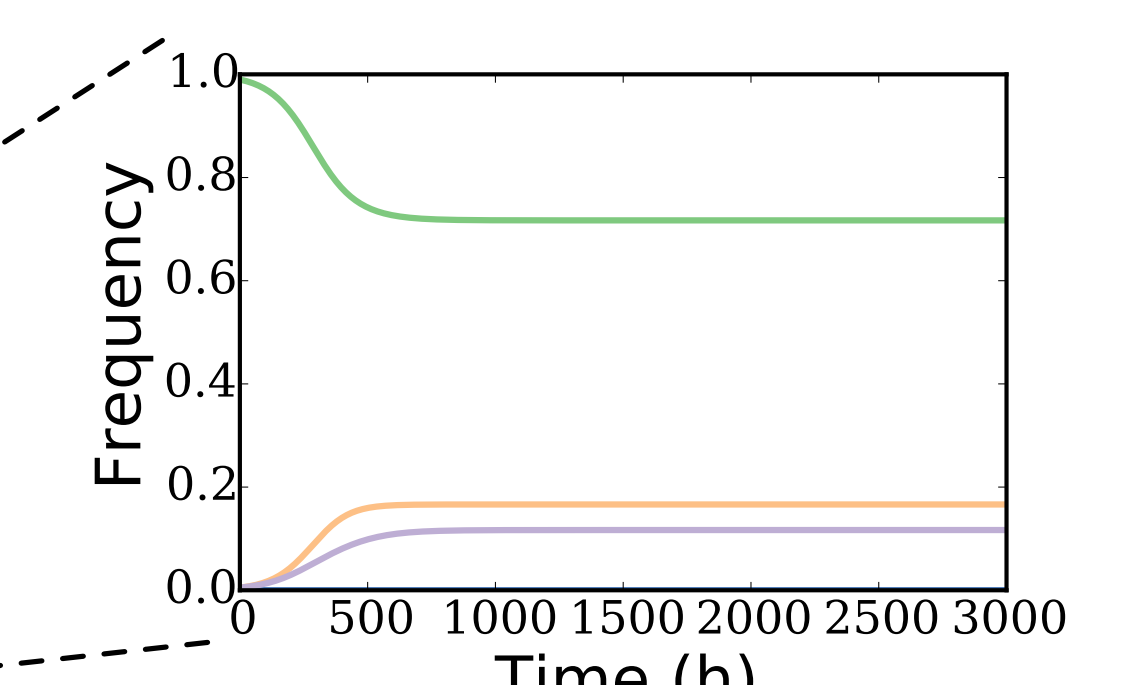

Time (h)

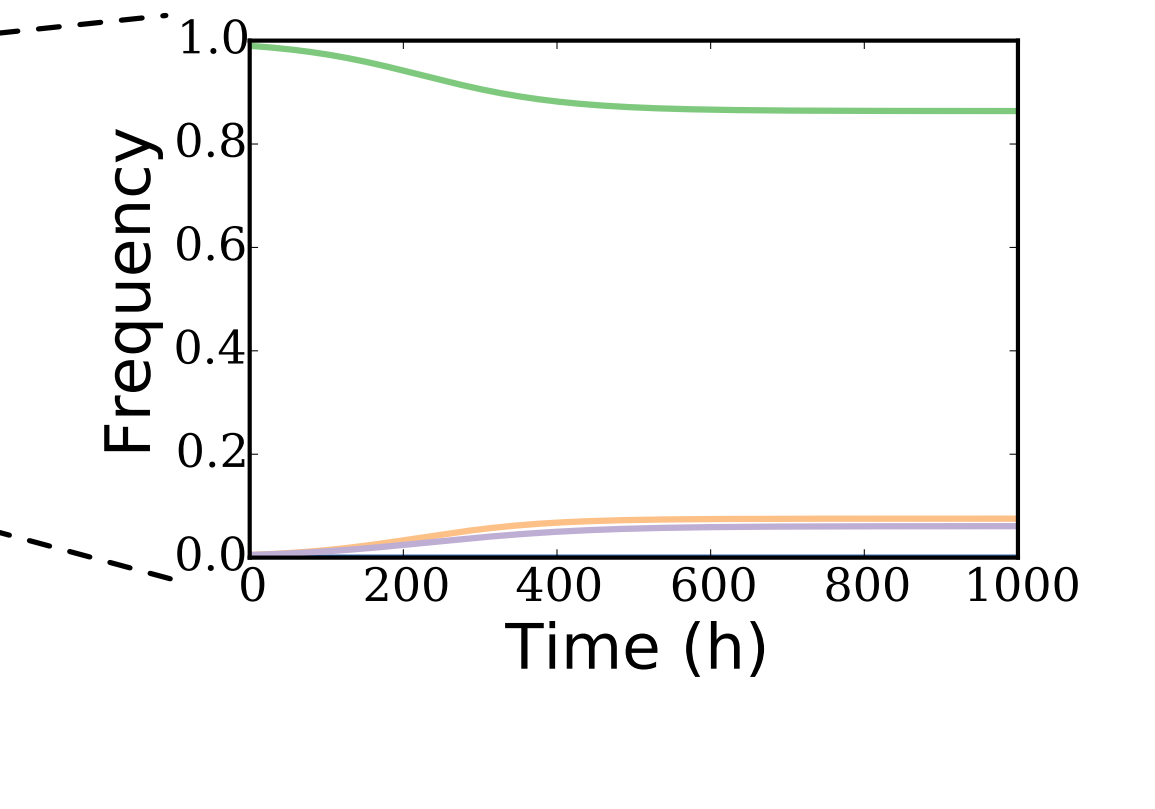

